Supporting Information for

\title{
Evolution of Pyrrolidine-Type Asymmetric Organocatalysts by Click Chemistry
}

Sanzhong Luo, ${ }^{* \dagger}$ Hui Xu, ${ }^{\dagger}$ Xueling Mi, ${ }^{\dagger}$ Jiuyuan Li, ${ }^{\dagger}$ Xiaoxi Zheng, ${ }^{\dagger}$ and Jin-Pei Cheng $*^{\dagger *}$

Beijing National Laboratory for Molecular Sciences (BNLMS), Center for Molecular Science, Institute of Chemistry, Chinese Academy of Sciences, Beijing, 100080, China and Department of Chemistry, State Key Laboratory of elemento-organic Chemistry, Nankai University, Tianjin, 300071, China.

luosz@iccas.ac.cn, chengjp@mail.most.gov.cn

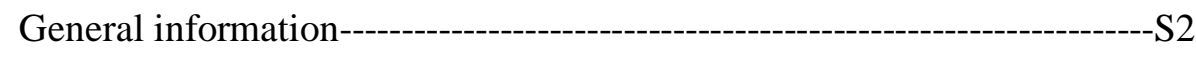

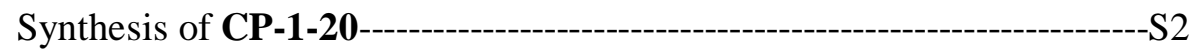

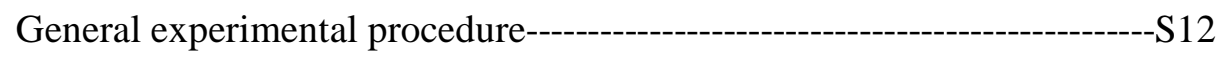

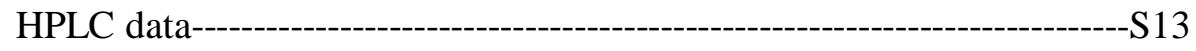

NMR spectra for new compounds-----------------------------------------------S16

${ }^{1} \mathrm{H}$ NMR spectra for Michael addition products--------------------------------S36 
General Information: Commercial reagents were used as received, unless otherwise stated. Chemical shifts are reported in ppm from tetramethylsilane with the solvent resonance as the internal standard. The following abbreviations were used to designate chemical shift mutiplicities: $\mathrm{s}$ $=$ singlet, $\mathrm{d}=$ doublet, $\mathrm{t}=$ triplet, $\mathrm{q}=$ quartet, $\mathrm{h}=$ heptet, $\mathrm{m}=$ multiplet, $\mathrm{br}=$ broad. All first-order splitting patterns were assigned on the basis of the appearance of the multiplet. Splitting patterns that could not be easily interpreted are designated as multiplet (m) or broad (br). Mass spectra were obtained using fast-atom bombard (FAB) spectrometer or electrospray ionization (ESI) mass spectrometer. Optical rotations were measured using a $1 \mathrm{~mL}$ cell with a $1 \mathrm{dm}$ path length on a Perkin-Elmer 341 digital polarimeter and are reported as follows: $[\alpha]_{\mathrm{D}}{ }^{\mathrm{rt}}(c$ in $\mathrm{g}$ per $100 \mathrm{~mL}$ of solvent). HPLC analysis was performed using ChiralPak columns purchased.

\section{Method A:}

\section{Synthesis of chiral catalyst CP-2:}

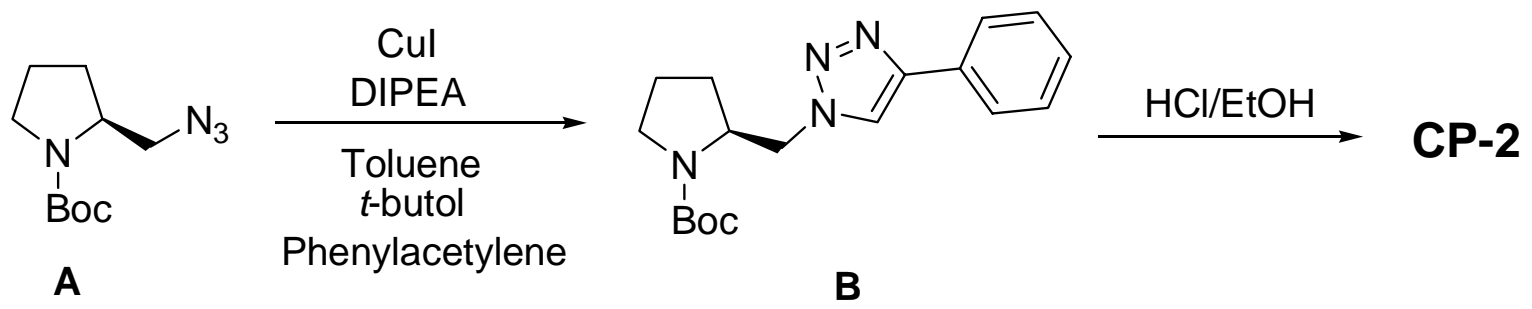

To a solution of $\mathbf{A}(226 \mathrm{mg}, 1 \mathrm{mmol})$ in toluene and $t$-butanol $(4 \mathrm{~mL}$ and $1 \mathrm{~mL})$ was added phenylacetylene (122 mg, $1.2 \mathrm{mmol})$, CuI (10 mg, $0.05 \mathrm{mmol})$ and DIPEA (170 $\mu \mathrm{L}, 2 \mathrm{mmol})$. The reaction mixture was stirred at rt overnight. After removal of the solvent under vacuo, the residue was purified by flash chromatograph on silica gel to afford $\mathbf{B}$ as white solid (314 mg, yield 96\%). ${ }^{1} \mathrm{H}$ NMR (300 MHz, $\left.\mathrm{CDCl}_{3}\right): \delta 1.37-1.65(10 \mathrm{H}, \mathrm{m}), 1.67-1.83(1 \mathrm{H}, \mathrm{m}), 1.89-2.07(2 \mathrm{H}, \mathrm{m}), 3.08-3.50$ $(2 \mathrm{H}, \mathrm{m}), 4.15(1 \mathrm{H}, \mathrm{s}), 4.37-4.79(2 \mathrm{H}, \mathrm{m}), 7.29-7.38(1 \mathrm{H}, \mathrm{m}), 7.38-7.48(2 \mathrm{H}, \mathrm{m}), 7.62-7.90(3 \mathrm{H}, \mathrm{m})$. 
Chiral product $\mathbf{B}$ was deprotected in $5 \mathrm{M} \mathrm{HCl}$ in ethanol to give the hydrogen chloride salts, which was subsequently dissolved in $\mathrm{CH}_{2} \mathrm{Cl}_{2}(5 \mathrm{~mL})$ and then treated with saturated $\mathrm{NaHCO}_{3}$ solution $(15 \mathrm{~mL})$. This mixture was stirred for 1 hour. The aqueous layer was extracted with $\mathrm{CH}_{2} \mathrm{Cl}_{2}$ ( $5 \mathrm{~mL} \times 3$ ). The combined organic layers were dried over anhydrous $\mathrm{Na}_{2} \mathrm{SO}_{4}$, and concentrated in vacuo after filtration to give essentially pure CP-2 as pale yellow solid $(301 \mathrm{mg}, 96 \%) .[\alpha]_{\mathrm{D}}{ }^{\mathrm{rt}}=+41^{\mathrm{o}}$ $\left(\mathrm{c}=1.0, \mathrm{CH}_{3} \mathrm{OH}\right) ;{ }^{1} \mathrm{H}$ NMR $\left(300 \mathrm{MHz}, \mathrm{CDCl}_{3}\right): \delta 1.35-1.52(1 \mathrm{H}, \mathrm{m}), 1.58-1.83(3 \mathrm{H}, \mathrm{m}), 1.85-1.99$ $(1 \mathrm{H}, \mathrm{m}), 2.89(2 \mathrm{H}, \mathrm{t}, J=6.6 \mathrm{~Hz}), 3.51-3.64(1 \mathrm{H}, \mathrm{m}), 4.11-4.21(1 \mathrm{H}, \mathrm{dd}, J=7.9 \mathrm{~Hz}, 7.7 \mathrm{~Hz}, 13.8 \mathrm{~Hz})$, 4.35-4.44 (1H, dd, J=4.5 Hz, 4.3 Hz, 13.4 Hz), 7.21-7.30 (1H, t, J= 7.5 Hz), 7.35 (2H, t, $J=7.4 \mathrm{~Hz})$, $7.77(2 \mathrm{H}, \mathrm{t}, J=7.3), 7.86(1 \mathrm{H}, \mathrm{s}) ;{ }^{13} \mathrm{C} \mathrm{NMR}\left(\mathrm{CDCl}_{3}, 75 \mathrm{MHz}\right): \delta 25.5,29.1,46.6,55.5,58.0,120.5$, 125.7, 128.0, 128.8, 130.7, 147.5; HRMS for $\mathrm{C}_{13} \mathrm{H}_{17} \mathrm{~N}_{4}{ }^{+}\left(\mathrm{M}+1^{+}\right)$, calcd. 229.1448, found 229.1446.

\section{Method B:}

\section{Synthesis of chiral catalyst CP-2:}

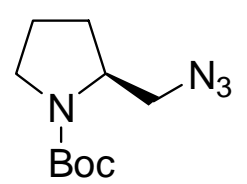

A

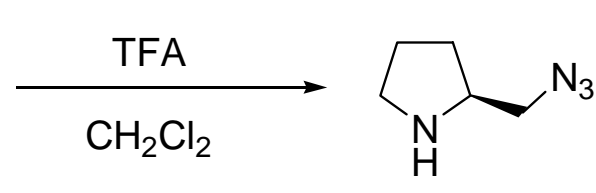

CP-1

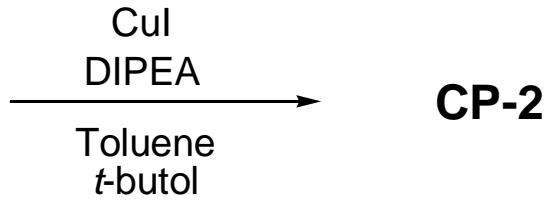

Phenylacetylene

To a solution of $\mathbf{A}(452 \mathrm{mg}, 2 \mathrm{mmol})$ in $\mathrm{CH}_{2} \mathrm{Cl}_{2}(5 \mathrm{~mL})$ was added dropwise TFA $(5 \mathrm{~mL})$ at $0^{\circ} \mathrm{C}$.

The mixture was warmed to room temperature and stirred overnight. After removal of the organic solvents under vacuo, the residue was dissolved in $\mathrm{CH}_{2} \mathrm{Cl}_{2}(5 \mathrm{~mL})$ and then treated with saturated $\mathrm{NaHCO}_{3}$ solution $(15 \mathrm{~mL})$ for 1 hour at rt. The aqueous layer was extracted with $\mathrm{CH}_{2} \mathrm{Cl}_{2}$ three times ( $5 \mathrm{~mL} \times 3$ ) and the combined extracts were dried over anhydrous $\mathrm{Na}_{2} \mathrm{SO}_{4}$. Concentration in vacuo after filtration gave CP-1 as yellow oil (438 mg, 97\%). $[\alpha]_{\mathrm{D}}{ }^{\mathrm{rt}}=-32^{\circ}\left(\mathrm{c}=0.75, \mathrm{CHCl}_{3}\right) \quad{ }^{1} \mathrm{H} \mathrm{NMR}(300$ $\left.\mathrm{MHz}, \mathrm{CDCl}_{3}\right): \delta$ 1.35-1.50 $(1 \mathrm{H}, \mathrm{m}), 1.66-2.00(3 \mathrm{H}, \mathrm{m}), 2.44-2.61(1 \mathrm{H}, \mathrm{m}), 2.86-3.04(2 \mathrm{H}, \mathrm{m})$, 
3.17-3.39 (3H, m); ${ }^{13} \mathrm{C} \mathrm{NMR}\left(\mathrm{CDCl}_{3}, 75 \mathrm{MHz}\right): \delta 25.5,29.0,46.6,56.2,57.7$. HRMS for $\mathrm{C}_{5} \mathrm{H}_{11} \mathrm{~N}_{4}{ }^{+}$ $\left(\mathrm{M}+1^{+}\right)$, calcd. 127.0984, found 127.0982.

To a solution of CP-1 (438mg) and phenylacetylene (245 mg, $2.4 \mathrm{mmol})$ in a mixed solvent of toluene $(8 \mathrm{~mL})$ and $t$-butanol $(2 \mathrm{~mL})$ was added $\mathrm{CuI}(20 \mathrm{mg}, 10 \mathrm{mmol})$ and DIPEA $(500 \mu \mathrm{L}, 6$ mmol). The reaction mixture was stirred at room temperature overnight. After removal of the solvents, the resulting residue was purified by flash chromatograph on silica gel to give $\mathbf{C P - 2}$ as yellow solid (365 mg, 83\%).

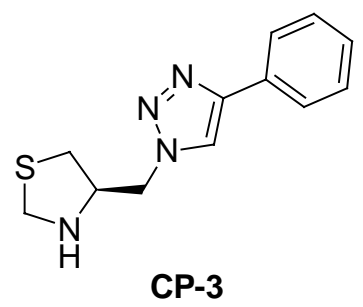

The title product was prepared according to method $\mathbf{A}$ as white solid (87\% yield). $[\alpha]_{\mathrm{D}}{ }^{\mathrm{rt}}=+10^{\circ}\left(\mathrm{c}=0.5, \mathrm{CH}_{3} \mathrm{OH}\right) ;{ }^{1} \mathrm{H} \mathrm{NMR}\left(300 \mathrm{MHz}, \mathrm{CDCl}_{3}\right)$ : 82.65-2.74 (1H, dd, $J=6.0 \mathrm{~Hz}, 6.0 \mathrm{~Hz}, 11.4 \mathrm{~Hz}), 2.99-3.07(1 \mathrm{H}, \mathrm{dd}, J=6.4$ Hz, 6.4 Hz, 10.6 Hz), 3.82-3.94 (1H, m), 4.22 (2H, s), 4.34-4.44 (1H, dd, J= 7.9 Hz, 7.9 Hz, $14.1 \mathrm{~Hz})$, 4.56-4.67 (1H, dd, J= 5.0 Hz, 5.1 Hz, $14.1 \mathrm{~Hz}), 7.29-7.37(1 \mathrm{H}, \mathrm{m}), 7.37-7.47(2 \mathrm{H}, \mathrm{m}), 7.79-7.88$ $(2 \mathrm{H}, \mathrm{m}), 7.89(1 \mathrm{H}, \mathrm{s}) ;{ }^{13} \mathrm{C} \mathrm{NMR}\left(\mathrm{CDCl}_{3}, 75 \mathrm{MHz}\right): \delta 35.1,50.4,52.4,63.0,119.6,124.7,127.2$ 127.8, 129.5, 146.8; HRMS for $\mathrm{C}_{12} \mathrm{H}_{15} \mathrm{~N}_{4} \mathrm{~S}^{+}\left(\mathrm{M}+1^{+}\right)$, calcd. 247.1012, found 247.1016.

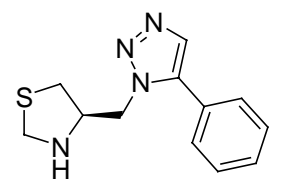

1,5-substituted triazole ring was formed following the published procedure. ${ }^{1}$ CP-4

To the dried flask containing a solution of EtMgBr $(2 \mathrm{mmol})$ in anhydrous THF (2 $\mathrm{mL}$ ) under a nitrogen atmosphere, phenylacetylene (204 mg, $2 \mathrm{mmol}$ ) was added dropwise at room temperature. After addition, the solution was heated to about $50{ }^{\circ} \mathrm{C}$ for $15 \mathrm{~min}$ and then cooled to room temperature. Neat (R)-tert-butyl 4-(azidomethyl)thiazolidine-3-carboxylate (244 mg, $1 \mathrm{mmol}$ ) was added dropwise. This reaction mixture was stirred under room temperature for $30 \mathrm{~min}$, then $50^{\circ} \mathrm{C}$ for $15 \mathrm{~min}$. The reaction was quenched with saturated aqueous $\mathrm{NH}_{4} \mathrm{Cl}$ and the 
products were extracted using $\mathrm{CH}_{2} \mathrm{Cl}_{2}(5 \mathrm{~mL} \times 3)$. The combined organic phase was dried over anhydrous sodium sulphate and concentrated by rotary evaporator under reduced pressure. The residue was purified by flash chromatograph on silica gel to give Boc-protected $\mathbf{C P}-\mathbf{4}$ as pale yellow oil (310mg, 90\% yield).

The Boc-protected CP-4 was deprotected using 4M $\mathrm{HCl}$ dioxane solution (5 mL). Concentration in vacuo afforded the hydrogen chlorides salts, which was subsequently neutralized in saturated $\mathrm{NaHCO}_{3}$ solution $(5 \mathrm{~mL})$. The aqueous solution was extracted with $\mathrm{CH}_{2} \mathrm{Cl}_{2}(10 \mathrm{~mL} \times 3)$. The combined extracts were dried over anhydrous sodium sulphate, and then concentrated in vacuo to give CP-4 as yellow oil (90\% yield). $[\alpha]_{\mathrm{D}}{ }^{\mathrm{rt}}=-10^{\mathrm{o}},\left(\mathrm{c}=0.6, \mathrm{CHCl}_{3}\right) ;{ }^{1} \mathrm{H} \mathrm{NMR}\left(300 \mathrm{MHz}, \mathrm{CDCl}_{3}\right): \delta$ $2.10(1 \mathrm{H}, \mathrm{s}), 2.47-2.56(1 \mathrm{H}, \mathrm{m}), 2.79-2.88(1 \mathrm{H}, \mathrm{m}), 3.72-3.86(1 \mathrm{H}, \mathrm{m}), 3.88-.394(1 \mathrm{H}, \mathrm{dd}, J=2.5 \mathrm{~Hz}$, $2.5 \mathrm{~Hz}, 9.6 \mathrm{~Hz}), 3.99-4.06(1 \mathrm{H}, \mathrm{dd}, J=2.8 \mathrm{~Hz}, 2.8 \mathrm{~Hz}, 9.6 \mathrm{~Hz}), 4.35-4.53(2 \mathrm{H}, \mathrm{m}), 7.33-7.42(2 \mathrm{H}, \mathrm{m})$, 7.42-7.52 (3H, m), 7.63-7.68 (1H, m); ${ }^{13} \mathrm{C} \mathrm{NMR}\left(\mathrm{CDCl}_{3}, 75 \mathrm{MHz}\right): \delta 36.0,49.0,53.3,64.0,126.9$, 129.0, 129.1, 129.6, 133.0, 138.6; HRMS for $\mathrm{C}_{12} \mathrm{H}_{15} \mathrm{~N}_{4} \mathrm{~S}^{+}\left(\mathrm{M}+1^{+}\right)$, calcd. 247.1012, found 247.1012.

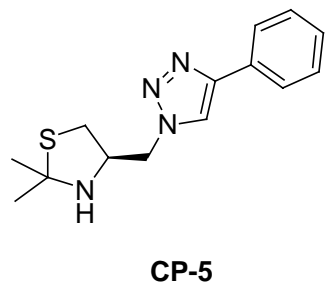

The title compound was prepared according to method $\mathbf{A}$ as white solid $(83 \%$ yield $) .[\alpha]_{\mathrm{D}}{ }^{\mathrm{rt}}=-120.4^{\mathrm{o}},\left(\mathrm{c}=0.5, \mathrm{CH}_{3} \mathrm{OH}\right) ;{ }^{1} \mathrm{H}$ NMR $(300 \mathrm{MHz}$, $\left.\mathrm{CDCl}_{3}\right): \delta 1.54(3 \mathrm{H}, \mathrm{s}), 1.64(3 \mathrm{H}, \mathrm{s}), 1.73-2.23(1 \mathrm{H}, \mathrm{br}), 2.80(1 \mathrm{H}, \mathrm{t}, J=9.8$

Hz), 3.15-3.23 $(1 \mathrm{H}, \mathrm{dd}, J=6.0 \mathrm{~Hz}, 5.8 \mathrm{~Hz}, 10.6 \mathrm{~Hz}), 3.80-4.02(1 \mathrm{H}, \mathrm{m}), 4.53-4.61(1 \mathrm{H}, \mathrm{dd}, J=6.8 \mathrm{~Hz}$, 6.6 Hz, 14.1 Hz), 4.67-4.79 (1H, dd, $J=4.9 \mathrm{~Hz}, 5.1 \mathrm{~Hz}, 13.9 \mathrm{~Hz}), 7.28-7.37(1 \mathrm{H}, \mathrm{m}), 7.37-7.49(2 \mathrm{H}$, m), 7.78-7.85 (2H, m), $7.91(1 \mathrm{H}, \mathrm{s}) ;{ }^{13} \mathrm{C} \mathrm{NMR}\left(\mathrm{CDCl}_{3}, 75 \mathrm{MHz}\right): \delta 30.6,31.6,38.4,51.6,62.1,74.2$, 119.6, 124.7, 127.3, 127.9, 129.4, 146.8; HRMS for $\mathrm{C}_{14} \mathrm{H}_{19} \mathrm{~N}_{4} \mathrm{~S}^{+}\left(\mathrm{M}+1^{+}\right)$, calcd. 275.1325, found 275.1329. 


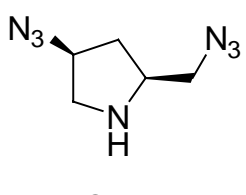

CP-6

To a stirred solution of $(2 S, 4 S)$-( tert-Butoxycarbonyl)-4-(p-toluenesulfony-loxy)-2-[(p-toluenesulfonyloxy)methyl]pyrrolidine $(1.44 \mathrm{~g}, 1.74 \mathrm{mmol})[\mathrm{J}$. Org. Chem. 1980, 45, 4728-4739] in DMF (15 mL) was added $\mathrm{NaN}_{3}$ in portions at rt. The reaction mixture was allowed to warm to $70{ }^{\circ} \mathrm{C}$ for 3 hours, and then $90{ }^{\circ} \mathrm{C}$ for 5 hours. After removal of solvent under reduced pressure, the residue was diluted in a mixture of $\mathrm{H}_{2} \mathrm{O}(50 \mathrm{~mL})$ and ethyl acetate $(15 \mathrm{~mL})$. The aqueous layer was extracted by ethyl acetate $(10 \mathrm{~mL} \times 3)$. The combined organic phase was dried over anhydrous sodium sulphate and concentrated by rotary evaporator. The residue was purified by flash chromatograph on silica gel to afford Boc-protected CP-6 as colorless oil (626 mg, 86\% yield).

Boc-protected CP-6 from the former step $(267 \mathrm{mg}, 1 \mathrm{mmol})$ was deprotected in a mixture of $\mathrm{CH}_{2} \mathrm{Cl}_{2}(2 \mathrm{~mL})$ and TFA $(2 \mathrm{~mL})$. Concentration in vacuo gave the TFA salts, which was subsequently neutralized in saturated $\mathrm{NaHCO}_{3}$ solution $(5 \mathrm{~mL})$. The aqueous solution was extracted with $\mathrm{CH}_{2} \mathrm{Cl}_{2}(10 \mathrm{~mL} \times 3)$. The combined organic layer was dried with anhydrous sodium sulphate and concentrated in vacuo to afford CP-6 as yellow oil $(166 \mathrm{mg}, 99 \%$ yield $) .[\alpha]_{\mathrm{D}}{ }^{\mathrm{rt}}=+18^{\mathrm{o}},(\mathrm{c}=0.3$, $\left.\mathrm{CHCl}_{3}\right) ;{ }^{1} \mathrm{H}$ NMR $\left(300 \mathrm{MHz}, \mathrm{CDCl}_{3}\right): \delta 1.52-1.62(1 \mathrm{H}, \mathrm{m}), 1.84(1 \mathrm{H}, \mathrm{s}), 2.17-2.30(1 \mathrm{H}, \mathrm{m})$, 2.27-3.11 (2H, m), 3.26-3.42 (3H, m), 3.98-4.08 (1H, m); ${ }^{13} \mathrm{C} \mathrm{NMR}\left(\mathrm{CDCl}_{3}, 75 \mathrm{MHz}\right): \delta 35.3,52.6$, 55.5, 57.3, 61.5; HRMS for $\mathrm{C}_{5} \mathrm{H}_{10} \mathrm{~N}_{7}^{+}\left(\mathrm{M}+1^{+}\right)$, calcd. 168.0992, found 168.0993.

The "click reaction" between Boc-protected CP-6 with phenylacetylene afforded simultaneously three products, i.e. the Boc-protected CP-7, CP-8 and CP-9 with 19\%, 14\%, 40\% yield, respectively. Those Boc-protected products were deprotected using the standard procedure to give the final products CP-7, CP-8 and CP-9. 


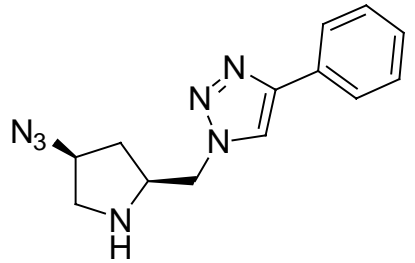

CP-7

Yellow solid. $[\alpha]_{\mathrm{D}}{ }^{\mathrm{rt}}=+38.5^{\circ},\left(\mathrm{c}=0.36, \mathrm{CHCl}_{3}\right) ;{ }^{1} \mathrm{H} \mathrm{NMR}(300 \mathrm{MHz}$, $\left.\mathrm{CDCl}_{3}\right): \delta 1.58-1.68(1 \mathrm{H}, \mathrm{m}), 2.06(1 \mathrm{H}, \mathrm{s}), 2.22-2.38(1 \mathrm{H}, \mathrm{m}), 2.96-3.20$ $(2 \mathrm{H}, \mathrm{m}), 3.64-3.78(1 \mathrm{H}, \mathrm{m}), 3.98-4.10(1 \mathrm{H}, \mathrm{m}), 4.26-4.39(1 \mathrm{H}, \mathrm{m})$, 4.42-4.58 (1H, m), 7.27-7.37 (1H, m), 7.37-7.47 (2H, t, J= 7.2 Hz), 7.78-7.87 (2H, d, $J=7.4 \mathrm{~Hz}), 7.90(1 \mathrm{H}, \mathrm{s}) ;{ }^{13} \mathrm{C} \mathrm{NMR}\left(\mathrm{CDCl}_{3}, 75 \mathrm{MHz}\right): \delta 35.3,52.2,55.1,57.1,61.1$, 120.7, 125.7, 128.1, 128.8, 130.6, 147.6; HRMS for $\mathrm{C}_{13} \mathrm{H}_{16} \mathrm{~N}_{7}{ }^{+}\left(\mathrm{M}+1^{+}\right)$, calcd. 270.1462, found 270.1463.

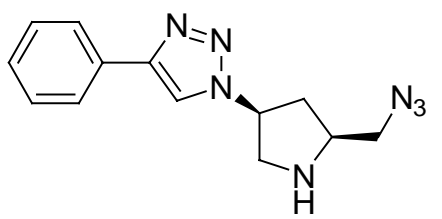

CP-8
Yellow solid. $[\alpha]_{\mathrm{D}}{ }^{\mathrm{rt}}=+16.0^{\circ},\left(\mathrm{c}=0.5, \mathrm{CHCl}_{3}\right) ;{ }^{1} \mathrm{H}$ NMR $(300$ $\left.\mathrm{MHz}, \mathrm{CDCl}_{3}\right): \delta 1.90-2.09(3 \mathrm{H}, \mathrm{m}), 2.58-2.69(1 \mathrm{H}, \mathrm{m}), 3.32-3.60(4 \mathrm{H}$, m), 5.15-5.25 (1H, m), 7.29-7.37 (1H, m), 7.38-7.48 (2H, t, J= 7.2 Hz),

$7.84(2 \mathrm{H}, \mathrm{d}, J=7.3 \mathrm{~Hz}), 8.01(1 \mathrm{H}, \mathrm{s}) ;{ }^{13} \mathrm{C} \mathrm{NMR}\left(\mathrm{CDCl}_{3}, 75 \mathrm{MHz}\right): \delta 36.6,53.6,55.1,57.8,60.6$, 118.1, 125.7, 128.2, 128.8, 130.6, 130.6, 148.1; HRMS for $\mathrm{C}_{13} \mathrm{H}_{16} \mathrm{~N}_{7}^{+}\left(\mathrm{M}+1^{+}\right)$, calcd. 270.1462, found 270.1465 .

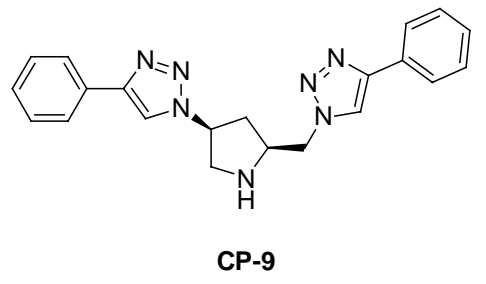

White solid. $[\alpha]_{\mathrm{D}}{ }^{\mathrm{rt}}=+16.7^{\mathrm{o}},\left(\mathrm{c}=0.48, \mathrm{CHCl}_{3}\right) ;{ }^{1} \mathrm{H} \mathrm{NMR}(300$ $\left.\mathrm{MHz}, \mathrm{CDCl}_{3}\right): \delta 2.05-2.17(2 \mathrm{H}, \mathrm{m}), 2.65-2.78(1 \mathrm{H}, \mathrm{m}), 3.29-3.38$ $(1 \mathrm{H}, \mathrm{dd}, J=3.6 \mathrm{~Hz}, 3.6 \mathrm{~Hz}, 11.5 \mathrm{~Hz}), 3.38-4.48(1 \mathrm{H}, \mathrm{dd}, J=6.4 \mathrm{~Hz}$, $6.4 \mathrm{~Hz}, 11.5 \mathrm{~Hz}), 3.81-3.92(1 \mathrm{H}, \mathrm{m}), 4.46-4.67(2 \mathrm{H}, \mathrm{m}), 5.11-5.23(1 \mathrm{H}, \mathrm{m}), 7.29-7.46(6 \mathrm{H}, \mathrm{m})$, 7.74-7.87 (5H, m), $7.93(1 \mathrm{H}, \mathrm{s}) ;{ }^{13} \mathrm{C} \mathrm{NMR}\left(\mathrm{CDCl}_{3}, 75 \mathrm{MHz}\right): \delta 35.2,52.5,53.2,56.7,59.3,117.1$, 119.9, 124.7, 127.1, 127.2, 127.8, 127.9, 129.4, 146.7, 147.1; HRMS for $\mathrm{C}_{21} \mathrm{H}_{22} \mathrm{~N}_{7}^{+}\left(\mathrm{M}+1^{+}\right)$, calcd. 372.1931, found 372.1931. 


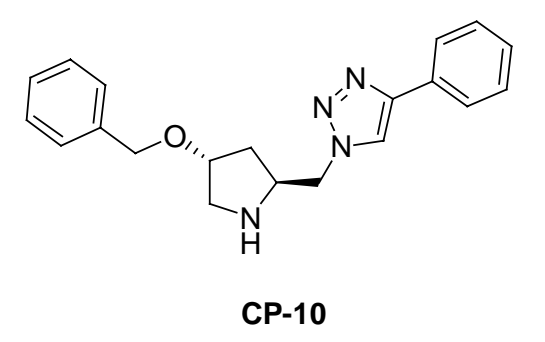

Prepared according to method A as colorless oil (20\% yield, $79 \%$ of the starting materials were recycled). $[\alpha]_{\mathrm{D}} \stackrel{\mathrm{rt}}{=}+6 \cdot 0^{\circ}$, $\left(\mathrm{c}=0.67, \mathrm{CHCl}_{3}\right) ;{ }^{1} \mathrm{H}$ NMR $\left(300 \mathrm{MHz}, \mathrm{CDCl}_{3}\right): \delta 1.39-1.58(1 \mathrm{H}$, m), $1.97-2.15(2 \mathrm{H}, \mathrm{m}), 2.75-2.85(1 \mathrm{H}, \mathrm{dd}, J=4.0 \mathrm{~Hz}, 4.2 \mathrm{~Hz}, 12.3$ Hz), 2.95-3.06 (1H, m), 3.72-3.84 (1H, m), 3.95-4.05 (1H, m), 4.10-4.20 (1H, dd, J= 7.4 Hz, 7.4 Hz, 14.1 Hz), 4.30-4.44 (3H, m), 7.13-7.28 (5H, m), 7.28-7.40 (3H, m), 7.72-7.78 (2H, m), 7.88 (1H, s); ${ }^{13} \mathrm{C} \mathrm{NMR}\left(\mathrm{CDCl}_{3}, 75 \mathrm{MHz}\right): \delta 34.6,51.1,51.3,54.1,55.8,69.7,78.8,119.6,124.7,126.5,126.6$, 126.7, 127.0, 127.4, 127.8, 129.7, 137.1, 146.5; HRMS for $\mathrm{C}_{20} \mathrm{H}_{23} \mathrm{~N}_{4} \mathrm{O}^{+}\left(\mathrm{M}+1^{+}\right)$, calcd. 335.1866, found 335.1867 .

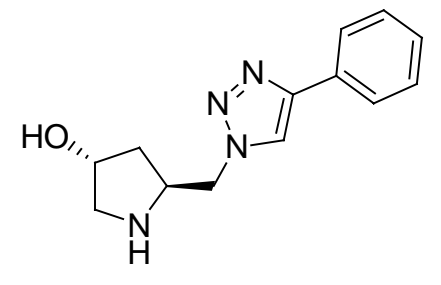

CP-11 (2S,4R)-tert-butyl 4-(benzyloxy)-2-((4-phenyl-1H-1,2,3-triazol-1-yl)methyl)pyrrolidine-1-carboxylate (515 mg, $1.19 \mathrm{mmol}$ ) was treated with $\mathrm{Pd} / \mathrm{C}(100 \mathrm{mg})$ and methanol $(15 \mathrm{~mL})$ under hydrogen. The reaction mixture was stirred at $60{ }^{\circ} \mathrm{C}$ until the substrate was completely consumed

by TLC. The mixture was filtered through Celite and the resulting solution was concentrated in vacuo to give Boc-CP-11 as colorless oil (125 mg, 32\% yield, starting materials were recycled in $65 \%$ yield).

The Boc- CP-11 obtained from former steps was deprotected in a mixture of $\mathrm{CH}_{2} \mathrm{Cl}_{2}(2 \mathrm{~mL})$ and TFA $(2 \mathrm{~mL})$. After removal of solvents in vacuo, the resulting TFA salt was subsequently neutralized in saturated $\mathrm{NaHCO}_{3}$ solution $(5 \mathrm{~mL})$. The aqueous solution was extracted with $\mathrm{CH}_{2} \mathrm{Cl}_{2}$ $(10 \mathrm{~mL} \times 3)$. The combined extracts were dried over anhydrous sodium sulphate. The organic solvent was concentrated in vacuo to afford $\mathbf{C P - 1 1}$ as colorless oil $\left(82 \mathrm{mg}, 92 \%\right.$ yield). $[\alpha]_{\mathrm{D}}{ }^{\mathrm{rt}}=+8.0^{\circ},(\mathrm{c}=0.25$, 
$\left.\mathrm{CHCl}_{3}\right) ;{ }^{1} \mathrm{H}$ NMR $\left(300 \mathrm{MHz}, \mathrm{CDCl}_{3}\right): \delta 1.57-1.72(1 \mathrm{H}, \mathrm{m}), 1.94-2.06(1 \mathrm{H}, \mathrm{m}), 2.06-2.33(3 \mathrm{H}, \mathrm{m})$, $2.95(1 \mathrm{H}, \mathrm{s}), 3.89-4.02(1 \mathrm{H}, \mathrm{m}), 4.22-4.32(1 \mathrm{H}, \mathrm{dd}, J=7.2 \mathrm{~Hz}, 7.5 \mathrm{~Hz}, 13.8 \mathrm{~Hz}), 4.37-4.60$ (2H, m), 7.29-7.38 (1H, m), 7.38-7.49 (2H, m), 7.78-7.88 (2H, m), $7.98(1 \mathrm{H}, \mathrm{s}) ;{ }^{13} \mathrm{C} \mathrm{NMR}\left(\mathrm{CDCl}_{3}, 75 \mathrm{MHz}\right)$ : $\delta 29.7,38.8,55.0,56.6,72.7,120.7,125.7,128.1,128.9,130.7,147.6$; HRMS for $\mathrm{C}_{13} \mathrm{H}_{17} \mathrm{~N}_{4} \mathrm{O}^{+}$ $\left(\mathrm{M}+1^{+}\right)$, calcd. 245.1397, found 245.1399.

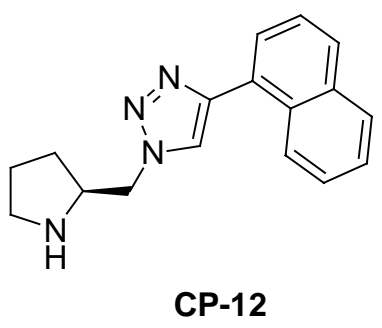

The title compound was prepared according to method $\mathbf{A}$ as yellow $(1 \mathrm{H}, \mathrm{dd}, J=7.9 \mathrm{~Hz}, 7.9 \mathrm{~Hz}, 13.6 \mathrm{~Hz}), 4.37-4.48(1 \mathrm{H}, \mathrm{dd}, J=4.5 \mathrm{~Hz}, 4.5 \mathrm{~Hz}, 13.4 \mathrm{~Hz}), 7.38-7.54(3 \mathrm{H}$, m), 7.66-7.73 $(1 \mathrm{H}, \mathrm{m}), 7.95(1 \mathrm{H}, \mathrm{s}), 8.37-8.43(1 \mathrm{H}, \mathrm{m}) ;{ }^{13} \mathrm{C} \mathrm{NMR}\left(\mathrm{CDCl}_{3}, 75 \mathrm{MHz}\right): \delta 24.9,28.6$, $46.0,54.7,57.4,123.1,124.9,125.0,125.5,126.1,126.7,127.7,127.9,128.3,130.6,133.4,146.0$; HRMS for $\mathrm{C}_{17} \mathrm{H}_{19} \mathrm{~N}_{4}^{+}\left(\mathrm{M}+1^{+}\right)$, calcd. 279.1604, found 279.1606.

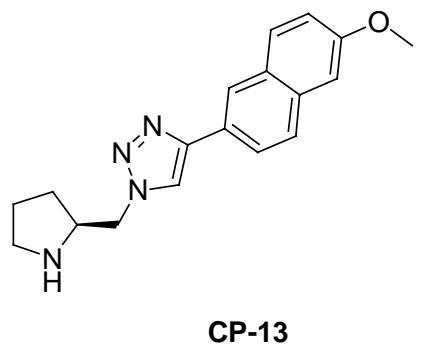

The title compound was prepared according to method $\mathbf{A}$ as white solid $(92 \%$ yield $) .[\alpha]_{\mathrm{D}}{ }^{\mathrm{rt}}=+10.3^{\circ},\left(\mathrm{c}=0.58, \mathrm{CHCl}_{3}\right) ;{ }^{1} \mathrm{H} \mathrm{NMR}(300 \mathrm{MHz}$, $\left.\mathrm{CDCl}_{3}\right): \delta 1.44-1.62(1 \mathrm{H}, \mathrm{m}), 1.62-1.88(2 \mathrm{H}, \mathrm{m}), 1.88-2.04(1 \mathrm{H}, \mathrm{m})$, 2.92-3.02 (1H, m), 3.56-3.76 (1H, br), 3.85-3.94 (3H, m), 4.22-4.37 (1H, m), 4.39-4.51 (1H, m), 7.06-7.14 (2H, m), 7.10-7.18 (2H, m), $7.75(2 \mathrm{H}, \mathrm{d}, J=8.5 \mathrm{~Hz}), 7.83-7.89(1 \mathrm{H}$, m), 8.00-8.06 (1H, m), 8.22 (1H, s); ${ }^{13} \mathrm{C} \mathrm{NMR}\left(\mathrm{CDCl}_{3}, 75 \mathrm{MHz}\right): \delta$ 24.4, 28.0, 45.5, 54.3, 57.2, 104.7, 118.2, 119.5, 123.1, 123.3, 126.3, 128.0, 128.7, 133.3, 146.7, 156.9; HRMS for $\mathrm{C}_{18} \mathrm{H}_{21} \mathrm{~N}_{4} \mathrm{O}^{+}$ $\left(\mathrm{M}+1^{+}\right)$, calcd. 309.1710, found 309.1708. 
The title compound was prepared according to method $\mathbf{A}$ as yellow oil $(92 \%$ yield $) .[\alpha]_{\mathrm{D}}{ }^{\mathrm{rt}}=$

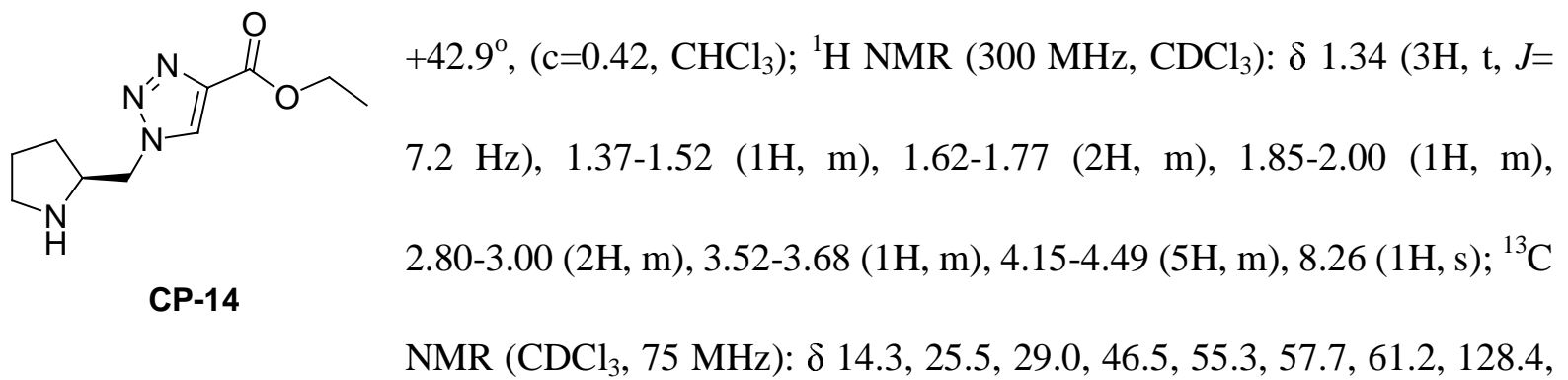
139.8, 160.8; HRMS for $\mathrm{C}_{10} \mathrm{H}_{17} \mathrm{~N}_{4} \mathrm{O}_{2}^{+}\left(\mathrm{M}+1^{+}\right)$, calcd. 225.1346, found 225.1346.

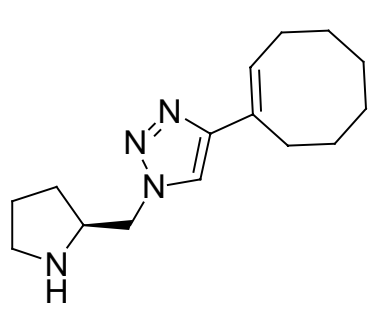

The title compound was prepared according to method $\mathbf{A}$ as yellow solid $(85 \%$ yield $) .[\alpha]_{\mathrm{D}}{ }^{\mathrm{rt}}=+14.5^{\mathrm{o}},\left(\mathrm{c}=0.83, \mathrm{CHCl}_{3}\right) ;{ }^{1} \mathrm{H}$ NMR $(300 \mathrm{MHz}$, $\left.\mathrm{CDCl}_{3}\right): \delta 1.31-1.61(9 \mathrm{H}, \mathrm{m}), 1.61-1.78(2 \mathrm{H}, \mathrm{m}), 1.78-1.94(1 \mathrm{H}, \mathrm{m}), 2.11$ CP-15 (1H, s), 2.16-2.30 (2H, m), $2.55(2 \mathrm{H}, \mathrm{t}, J=6.3 \mathrm{~Hz}), 2.85(2 \mathrm{H}, \mathrm{t}, J=6.6 \mathrm{~Hz})$, 3.44-3.58 (1H, m), 4.03-4.17 (1H, dd, J= 7.9 Hz, 7.7 Hz, 13.6 Hz), 4.24-4.35 (1H, dd, J= 4.5 Hz, 4.3 $\mathrm{Hz}, 13.8 \mathrm{~Hz}), 6.38(1 \mathrm{H}, \mathrm{t}, J=8.3 \mathrm{~Hz}), 7.53(1 \mathrm{H}, \mathrm{s}) ;{ }^{13} \mathrm{C} \mathrm{NMR}\left(\mathrm{CDCl}_{3}, 75 \mathrm{MHz}\right): \delta 24.4,25.0,25.6$, 25.7, 26.1, 27.8, 28.0, 29.0, 45.5, 54.3, 57.0, 118.7, 126.4, 129.4, 148.1; HRMS for $\mathrm{C}_{15} \mathrm{H}_{25} \mathrm{~N}_{4}{ }^{+}$ $\left(\mathrm{M}+1^{+}\right)$, calcd. 261.2074, found 261.2074.

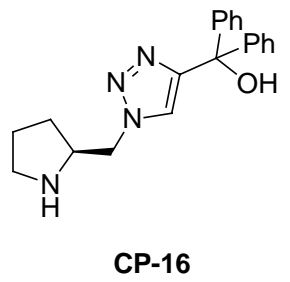

The title compound was prepared according to method $\mathbf{A}$ as yellow solid (90\% yield). $[\alpha]_{\mathrm{D}}{ }^{\mathrm{rt}}=+10.7^{\mathrm{o}},\left(\mathrm{c}=0.75, \mathrm{CHCl}_{3}\right) ;{ }^{1} \mathrm{H} \mathrm{NMR}\left(300 \mathrm{MHz}, \mathrm{CDCl}_{3}\right): \delta$ 1.23-1.39 (1H, m), 1.51-1.65 (2H, m), 1.70-1.83 (1H, m), $2.69(2 \mathrm{H}, \mathrm{t}, J=6.8 \mathrm{~Hz})$, 2.90-3.52 (3H, m), 3.96-4.08 (1H, dd, $J=7.7 \mathrm{~Hz}, 7.7 \mathrm{~Hz}, 13.6 \mathrm{~Hz}), 4.11-4.21(1 \mathrm{H}, \mathrm{dd}, J=4.9 \mathrm{~Hz}, 4.7$ $\mathrm{Hz}, 13.6 \mathrm{~Hz}), 6.85-7.45(11 \mathrm{H}, \mathrm{m}) ;{ }^{13} \mathrm{C} \mathrm{NMR}\left(\mathrm{CDCl}_{3}, 75 \mathrm{MHz}\right): \delta 25.2,29.0,46.3,55.2,57.7,76.5$, 123.6, 127.2, 127.3, 127.4, 127.8, 127.9, 146.1, 146.2, 153.9; $\mathrm{HRMS}$ for $\mathrm{C}_{20} \mathrm{H}_{23} \mathrm{~N}_{4} \mathrm{O}^{+}\left(\mathrm{M}+1^{+}\right)$, calcd. 335.1866, found 335.1866. 


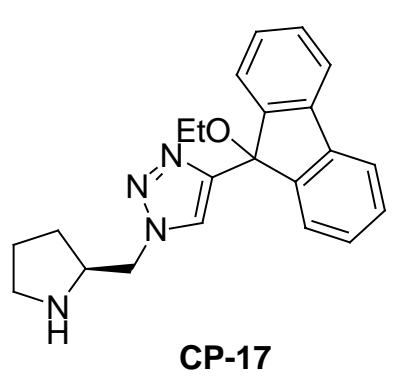

The title compound was prepared according to method $\mathbf{A}$ as yellow oil $(89 \%$ yield $) .[\alpha]_{\mathrm{D}}{ }^{\mathrm{rt}}=+4.8^{\circ},\left(\mathrm{c}=0.83, \mathrm{CHCl}_{3}\right) ;{ }^{1} \mathrm{H}$ NMR $(300 \mathrm{MHz}$, $\left.\mathrm{CDCl}_{3}\right): \delta 1.00(3 \mathrm{H}, \mathrm{t}, J=7.2 \mathrm{~Hz}), 1.24-1.39(1 \mathrm{H}, \mathrm{m}), 1.51-1.67(2 \mathrm{H}, \mathrm{m})$, 1.70-1.86 (1H, m), 1.90-2.60 (1H, br), 2.69-2.85 (2H, m), $2.97(2 \mathrm{H}, \mathrm{q}, J=$ $14.1 \mathrm{~Hz}), 3.34-3.48(1 \mathrm{H}, \mathrm{m}), 3.90-4.01(1 \mathrm{H}, \mathrm{m}), 4.15-4.24(1 \mathrm{H}, \mathrm{m})$, 7.14-7.35 (4H, m), $7.39(1 \mathrm{H}, \mathrm{s}), 7.47-7.55(1 \mathrm{H}, \mathrm{m}), 7.58(3 \mathrm{H}, \mathrm{d}, J=7.3 \mathrm{~Hz}) ;{ }^{13} \mathrm{C} \mathrm{NMR}\left(\mathrm{CDCl}_{3}, 75\right.$ MHz): $\delta 15.7,25.2,29.0,46.4,55.4,57.8,59.2,83.8,120.0,122.3,125.5,128.0,129.3,140.5,145.2$, 149.7; HRMS for $\mathrm{C}_{22} \mathrm{H}_{25} \mathrm{~N}_{4} \mathrm{O}^{+}\left(\mathrm{M}+1^{+}\right)$, calcd. 361.2023, found 361.2025.

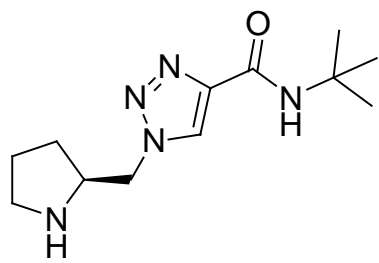

The title compound was prepared according to method $\mathbf{A}$ as white CP-18 2.00-2.40 (1H, br), 2.85-2.95 (2H, m), 3.47-.62 (1H, m), 4.15-4.25 (1H, dd, $J=7.7 \mathrm{~Hz}, 7.5 \mathrm{~Hz}, 13.6 \mathrm{~Hz}), 4.34-4.43(1 \mathrm{H}, \mathrm{dd}, J=4.7 \mathrm{~Hz}, 4.7 \mathrm{~Hz}, 13.6 \mathrm{~Hz}), 6.99$ (1H, s), 8.15 $(1 \mathrm{H}, \mathrm{s}) ;{ }^{13} \mathrm{C} \mathrm{NMR}\left(\mathrm{CDCl}_{3}, 75 \mathrm{MHz}\right): \delta 24.5,27.9,28.0,45.5,50.4,54.6,56.7,124.8,143.1,158.5$; HRMS for $\mathrm{C}_{12} \mathrm{H}_{22} \mathrm{~N}_{5} \mathrm{O}^{+}\left(\mathrm{M}+1^{+}\right)$, calcd. 252.1819, found 252.1820.

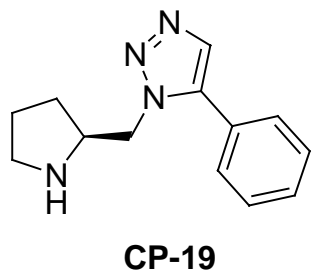

CP-19 was prepared following the similar procedure with that of CP-4 to give a yellow oil $(84 \%$ yield $) .[\alpha]_{\mathrm{D}}{ }^{\mathrm{rt}}=+3.4^{\mathrm{o}},\left(\mathrm{c}=0.58, \mathrm{CHCl}_{3}\right) ;{ }^{1} \mathrm{H} \mathrm{NMR}(300$ $\left.\mathrm{MHz}, \mathrm{CDCl}_{3}\right): \delta$ 1.28-1.43 (1H, m), 1.61-1.76 (2H, m), 1.76-1.89 (1H, m), 1.96-2.33 (1H, br), $2.88(2 \mathrm{H}, \mathrm{t}, J=6.6 \mathrm{~Hz}), 3.54-3.78(1 \mathrm{H}, \mathrm{m}), 4.14-4.35(1 \mathrm{H}, \mathrm{m}), 7.38-7.54(5 \mathrm{H}, \mathrm{m})$, $7.67(1 \mathrm{H}, \mathrm{s}) ;{ }^{13} \mathrm{C} \mathrm{NMR}\left(\mathrm{CDCl}_{3}, 75 \mathrm{MHz}\right): \delta 24.1,28.1,45.2,51.9,56.8,126.2,128.0,128.1,128.4$, 131.9, 137.3; HRMS for $\mathrm{C}_{13} \mathrm{H}_{17} \mathrm{~N}_{4}^{+}\left(\mathrm{M}+1^{+}\right)$, calcd. 229.1448, found 229.1447. 


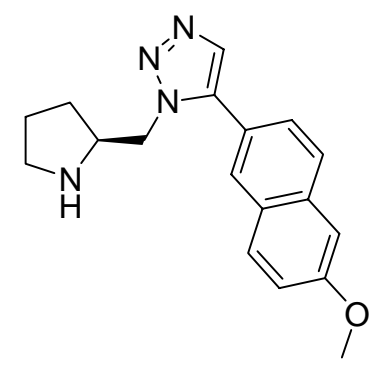

CP-20

CP-20 was prepared following the similar procedure with that of CP-4 to give a yellow oil $(63 \%$ yield $) .[\alpha]_{\mathrm{D}}{ }^{\mathrm{rt}}=-3.0^{\mathrm{o}},\left(\mathrm{c}=0.67, \mathrm{CHCl}_{3}\right) ;{ }^{1} \mathrm{H} \mathrm{NMR}$ $\left(300 \mathrm{MHz}, \mathrm{CDCl}_{3}\right): \delta 1.31-1.45(1 \mathrm{H}, \mathrm{m}), 1.61-1.77(2 \mathrm{H}, \mathrm{m}), 1.77-1.90(1 \mathrm{H}$, m), 1.94-2.37 (1H, br), $2.91(2 \mathrm{H}, \mathrm{t}, J=6.8 \mathrm{~Hz}), 3.61-3.78(1 \mathrm{H}, \mathrm{m}), 3.95(3 \mathrm{H}$, s), 4.20-4.40 (2H, m), 7.11-7.25 (2H, m), $7.48(1 \mathrm{H}, \mathrm{d}, J=8.1 \mathrm{~Hz}), 7.70-7.86$

(3H, m), $7.90(1 \mathrm{H}, \mathrm{s}) ;{ }^{13} \mathrm{C} \mathrm{NMR}\left(\mathrm{CDCl}_{3}, 75 \mathrm{MHz}\right): \delta 24.1,28.1,45.3,52.0,54.4,56.8,104.6,118.9$, 121.0, 125.6, 126.6, 127.5, 128.7, 132.0, 133.6, 137.5, 157.6; HRMS for $\mathrm{C}_{18} \mathrm{H}_{21} \mathrm{~N}_{4} \mathrm{O}^{+}\left(\mathrm{M}+1^{+}\right)$, calcd. 309.1710, found 309.1710.

Procedure for the Michael reaction: Nitrostyrene (37 mg, $0.25 \mathrm{mmol})$ and CP-2 (12 mg, 10 mol\%) were mixed with cyclohexanone $(0.5 \mathrm{~mL}, 5 \mathrm{mmol})$ in the presence of TFA $(0.00625 \mathrm{mmol}$, $0.2 \mu \mathrm{L}$ ) at room temperature (Bulk solution of TFA in cyclohexanone was freshly prepared and employed in the reaction, $20 \mu \mathrm{L}$ TFA in $50 \mathrm{~mL}$ of cyclohexanone). The homogeneous reaction mixture was stirred at room temperature for $18 \mathrm{~h}$. The reaction mixture was directly loaded onto silica gel column to afford the Michael adduct $1(61 \mathrm{mg}, 99 \%)$ as white solid: $[\alpha]_{\mathrm{D}}{ }^{\mathrm{rt}}=-15.2^{\circ}(\mathrm{c}=0.5$, $\mathrm{CH}_{3} \mathrm{OH}$ ), syn/anti=49:1 (by ${ }^{1} \mathrm{H}$ NMR), 92\% ee (by HPLC on a chiral phase chiralpak AD-H column, $\lambda=254 \mathrm{~nm}, i \mathrm{PrOH} / \mathrm{hexane} 10: 90,20^{\circ} \mathrm{C}, 0.5 \mathrm{~mL} \mathrm{~min}^{-1} ; t_{R}=22.7 \mathrm{~min}$ (minor), $29.4 \mathrm{~min}$ (major)). All the Michael addition products are known compounds. ${ }^{2-5}$

\section{HPLC conditions:}


$N_{(=)}^{(1)} N_{2}$<smiles>O=C1CCCC[C@H]1C[N+](=O)[O-]</smiles>
(major). (major).

The enantiomeric excess was determined by HPLC with an AD-H column at 254 $\mathrm{nm}$ (2-propanol: hexane $=10: 90), 25{ }^{\circ} \mathrm{C}, 0.5 \mathrm{~mL} / \mathrm{min} ; t_{R}=22.7 \mathrm{~min}$ (minor), $29.4 \mathrm{~min}$

The enantiomeric excess was determined by HPLC with an AD-H column at 254 $\mathrm{nm}$ (2-propanol: hexane $=10: 90), 20^{\circ} \mathrm{C}, 0.5 \mathrm{~mL} / \mathrm{min} ; t_{R}=27.4 \mathrm{~min}$ (minor), $41.6 \mathrm{~min}$<smiles>O=C1CCCCC1C(C[N+](=O)[O-])c1ccccc1Cl</smiles>

The enantiomeric excess was determined by HPLC with an AD-H column at 254 nm (2-propanol: hexane $=10: 90), 20^{\circ} \mathrm{C}, 0.5 \mathrm{~mL} / \mathrm{min} ; t_{R}=27.4 \mathrm{~min}$ (minor), $41.6 \mathrm{~min}$ (major).<smiles>O=C1CCCCC1C[N+](=O)[O-]</smiles>

The enantiomeric excess was determined by HPLC with an AD-H column at 254 $\mathrm{nm}$ (2-propanol: hexane $=10: 90), 20{ }^{\circ} \mathrm{C}, 0.5 \mathrm{~mL} / \mathrm{min} ; t_{R}=27.4 \mathrm{~min}$ (minor), $41.6 \mathrm{~min}$ (major).<smiles>O=C1CCCCC1C[N+](=O)[O-]</smiles>

The enantiomeric excess was determined by HPLC with an AD-H column at $254 \mathrm{~nm}$ (2-propanol: hexane $=10: 90), 20{ }^{\circ} \mathrm{C}, 0.5 \mathrm{~mL} / \mathrm{min} ; t_{R}=21.9 \mathrm{~min}$ (minor), 38.3 $\min ($ major).<smiles>Cc1ccc(C(C[N+](=O)[O-])C2CCCCC2=O)cc1</smiles>

The enantiomeric excess was determined by HPLC with an AD-H column at $254 \mathrm{~nm}$ (2-propanol: hexane $=10: 90), 20^{\circ} \mathrm{C}, 0.5 \mathrm{~mL} / \mathrm{min} ; t_{R}=18.0 \mathrm{~min}$ (minor), 23.5 $\min$ (major).<smiles>COc1ccc(C(C[N+](=O)[O-])C2CCCCC2=O)cc1</smiles>

The enantiomeric excess was determined by HPLC with an AD-H column at $254 \mathrm{~nm}$ (2-propanol: hexane=20:80), $20^{\circ} \mathrm{C}, 0.5 \mathrm{~mL} / \mathrm{min} ; t_{R}=19.1 \mathrm{~min}$ (minor), 23.9 
$\min$ (major).

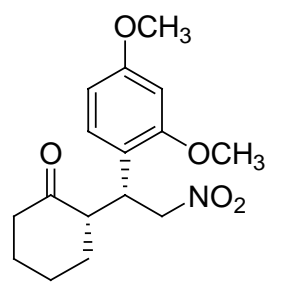

The enantiomeric excess was determined by HPLC with an AD-H column at $254 \mathrm{~nm}$ (2-propanol: hexane $=20: 80), 20^{\circ} \mathrm{C}, 0.5 \mathrm{~mL} / \mathrm{min} ; t_{R}=17.4 \mathrm{~min}$ (minor), $18.9 \mathrm{~min}$ (major). min<smiles>O=C1CCCC[C@H]1C[C@H](c1ccc2c(c1)OCO2)[N+](=O)[O-]</smiles>

The enantiomeric excess was determined by HPLC with an AD-H column at $254 \mathrm{~nm}$ (2-propanol: hexane=20:80), $20{ }^{\circ} \mathrm{C}, 0.5 \mathrm{~mL} / \mathrm{min} ; t_{R}=23.2 \mathrm{~min}$ (minor), 24.7 min (major).<smiles>O=C1CCC[C@H]1[C@H](C[N+](=O)[O-])c1ccccc1</smiles>

The enantiomeric excess was determined by HPLC with an AD-H column at $254 \mathrm{~nm}$ (2-propanol: hexane $=20: 80), 20^{\circ} \mathrm{C}, 0.5 \mathrm{~mL} / \mathrm{min} ; t_{R}=13.7 \mathrm{~min}$ (anti, major), $15.1 \mathrm{~min}$ (anti, minor), $16.2 \mathrm{~min}$ (syn, minor), $20.5 \min$ (syn, major).<smiles>CC(=O)CC(C[N+](=O)[O-])c1ccccc1</smiles>

The enantiomeric excess was determined by HPLC with an AD-H column at $254 \mathrm{~nm}$ (2-propanol: hexane=20:80), $20^{\circ} \mathrm{C}, 0.5 \mathrm{~mL} / \mathrm{min} ; t_{R}=14.5 \mathrm{~min}$ (minor), $15.4 \min$ (major)<smiles>CC(C)C(C=O)C(C[N+](=O)[O-])c1ccccc1</smiles>

The enantiomeric excess was determined by HPLC with an AD-H column at $281 \mathrm{~nm}$ (2-propanol: hexane=3:97), $20{ }^{\circ} \mathrm{C}, 0.5 \mathrm{~mL} / \mathrm{min} ; t_{R}=17.9 \mathrm{~min}$ (major), 21.0 $\min ($ minor)

\section{Reference:}

[1] Krasinski, A.; Fokin V. V.; Sharpless, K. B. Org. Lett. 2004, 6, 1237. 
[2] Luo, S.; Mi, X.; Zhang, L.; Liu, S.; Xu, H.; Cheng, J.-P. Angew. Chem. Int. Ed. 2006, 45, 3093

[3] (a) Ishii, T.; Fujioka, S.; Sekiguchi, Y.; Kotsuki, H. J. Am. Chem. Soc. 2004, 126, 9558-9559; (b) Betancort, J. M.; Sakthivel, K.; Thayumanavan, R.; Tanaka, F.; Barbas, C. F. III, Synthesis 2004, 1509-1521. (c) List, B.; Pojarliev, P.; J. Martin, H. Org. Lett. 2001, 3, 2423-2425.

[4] (a) Cobb, A. J. A.; Longbottom, D. A.; Shaw, D. M.; Ley, S. V. Chem. Commun. 2004, 1808-1809; (b) Cobb, A. J. A.; Shaw, D. M.; Longbottom, D. A.; Gold, J. B.; Ley, S. V. Org. Biomol. Chem. 2005, 3, 84-96;

[5] (a) Wang, W.; Wang, J.; Li, H. Angew. Chem. Int. Ed. 2005, 44, 1369; (b) Hayashi, Y.; Gotoh, H.; Hayashi, T.; Shoji, M. Angew. Chem. Int. Ed. 2005, 44, 4212-4215. 


\section{NMR spectra for the clicked catalysts}
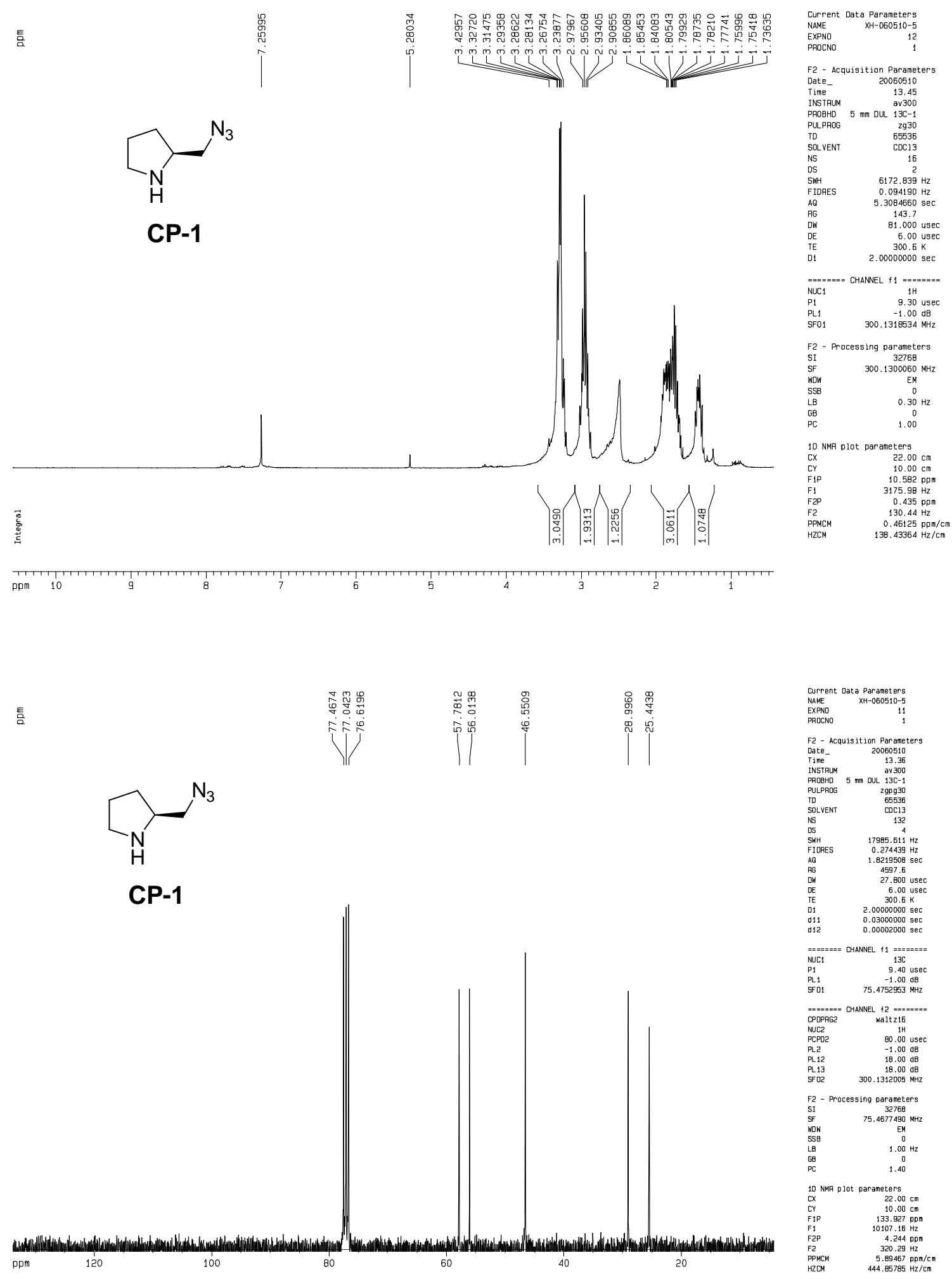

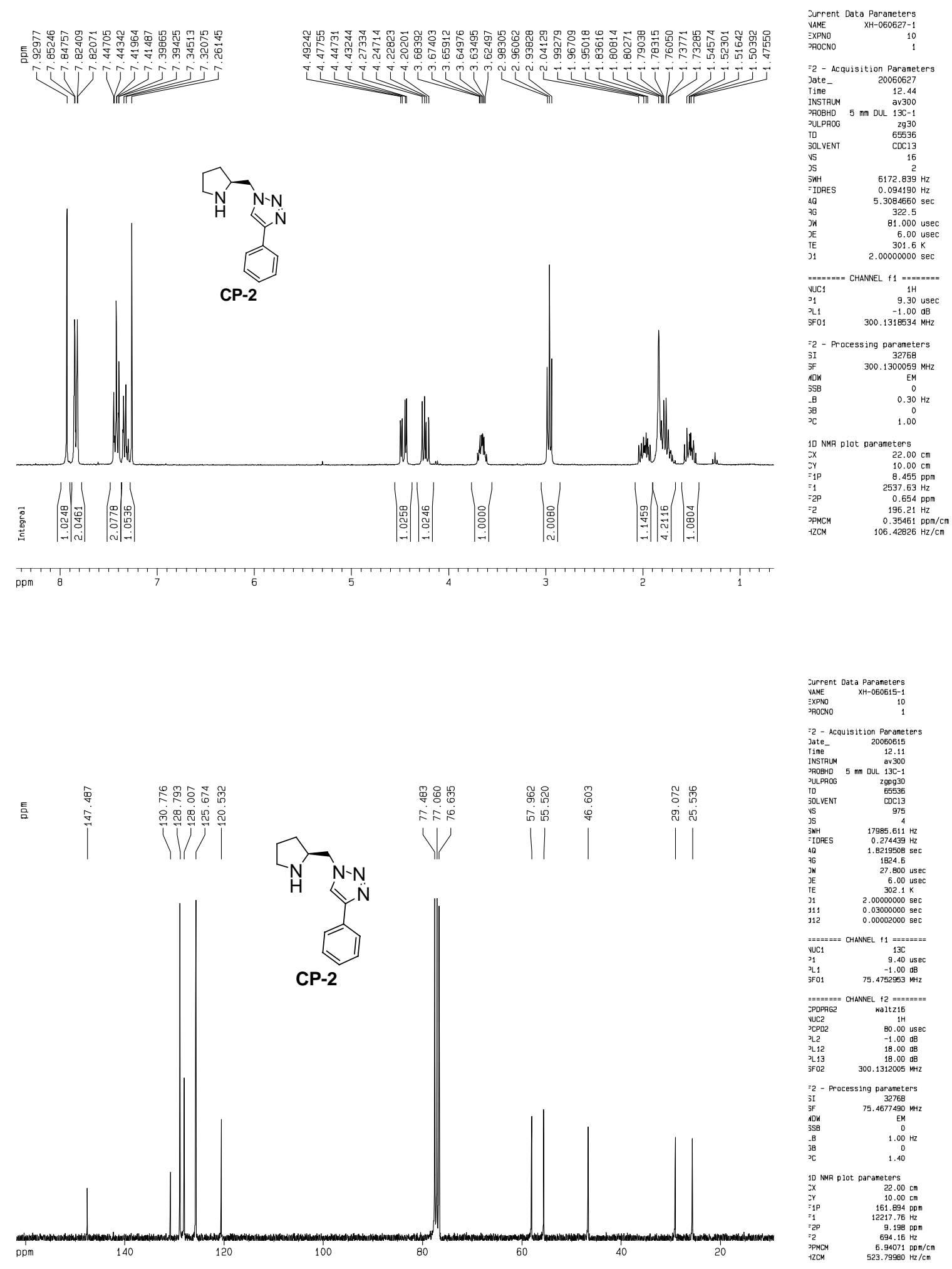

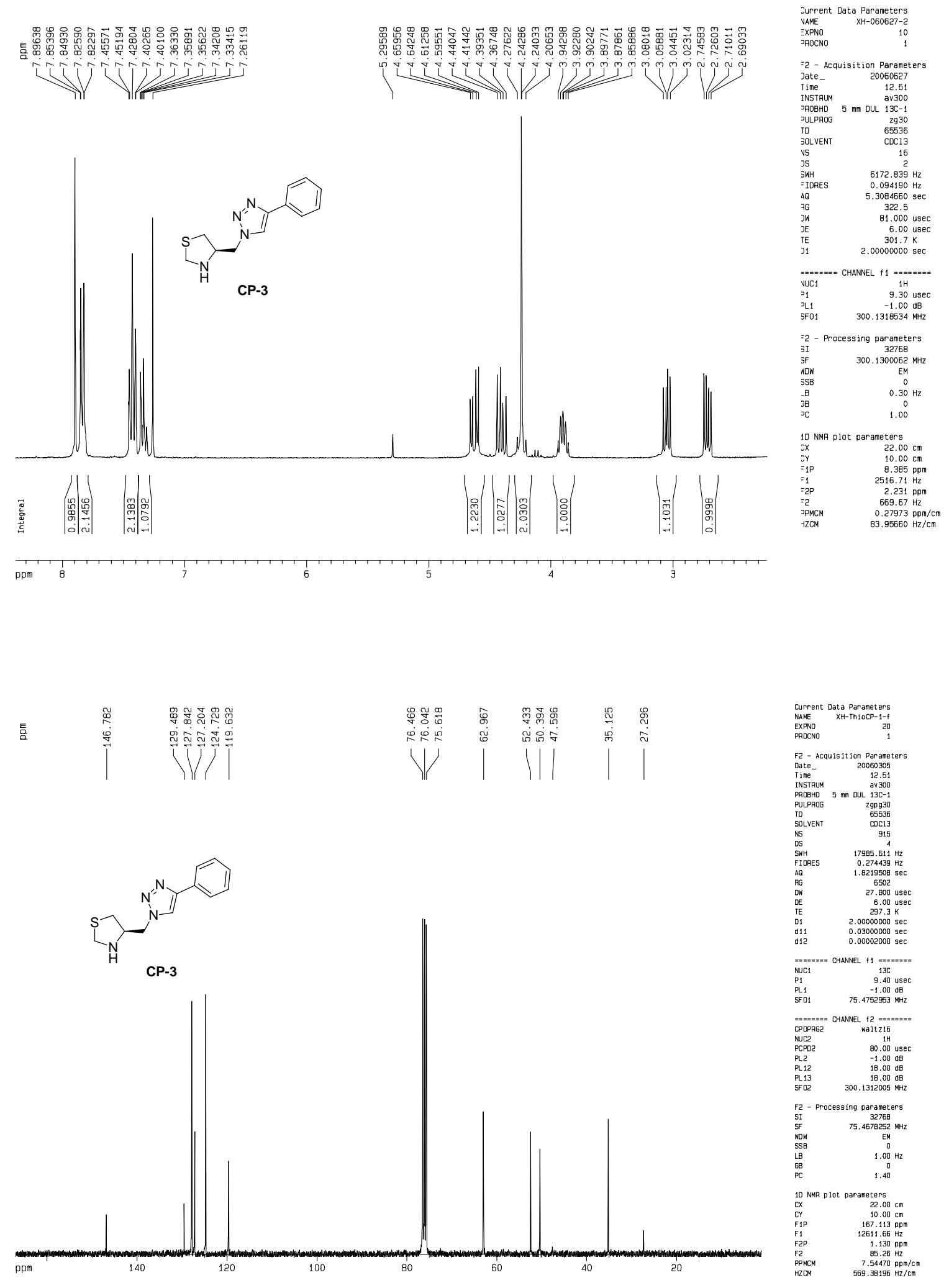

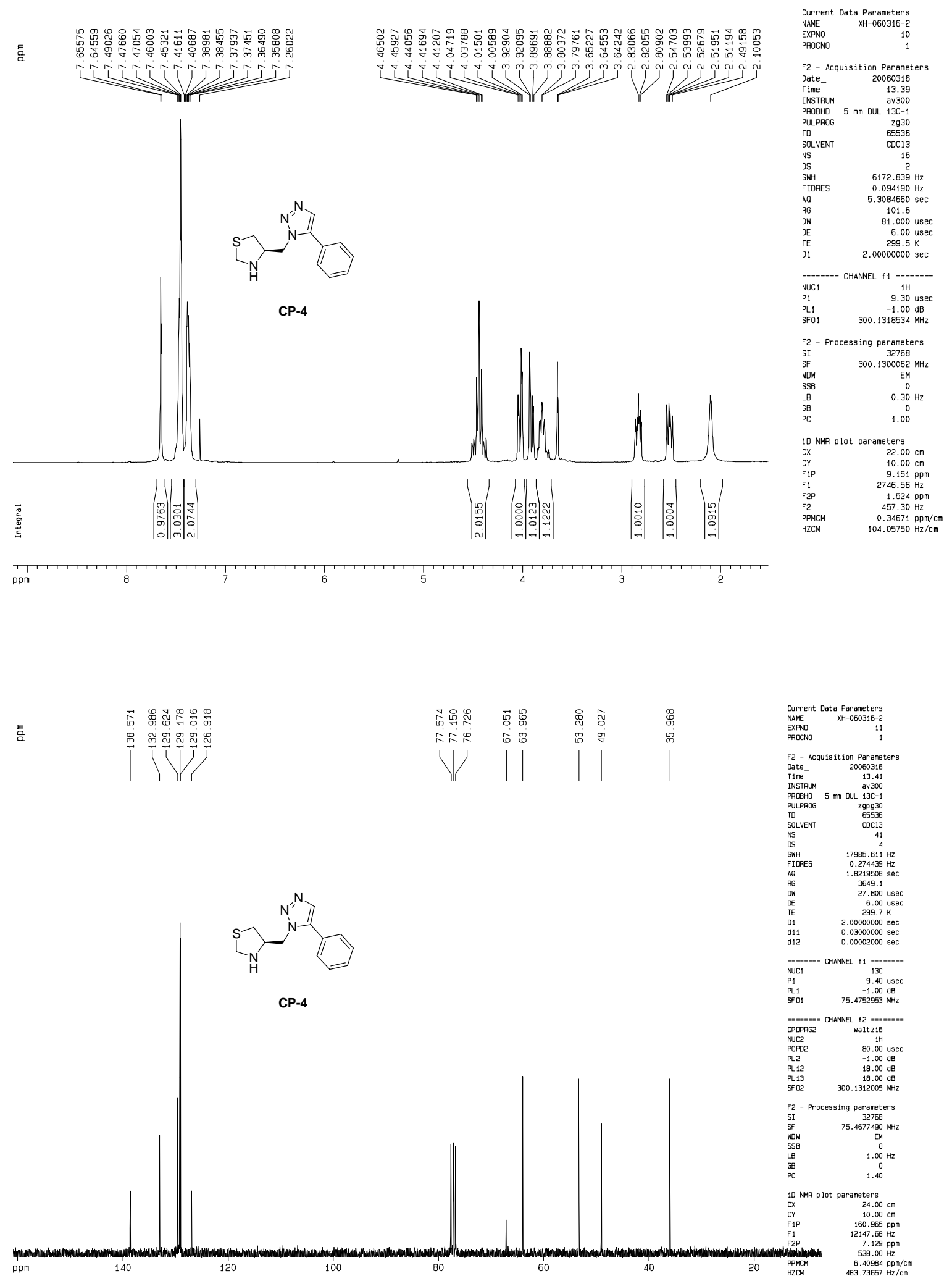

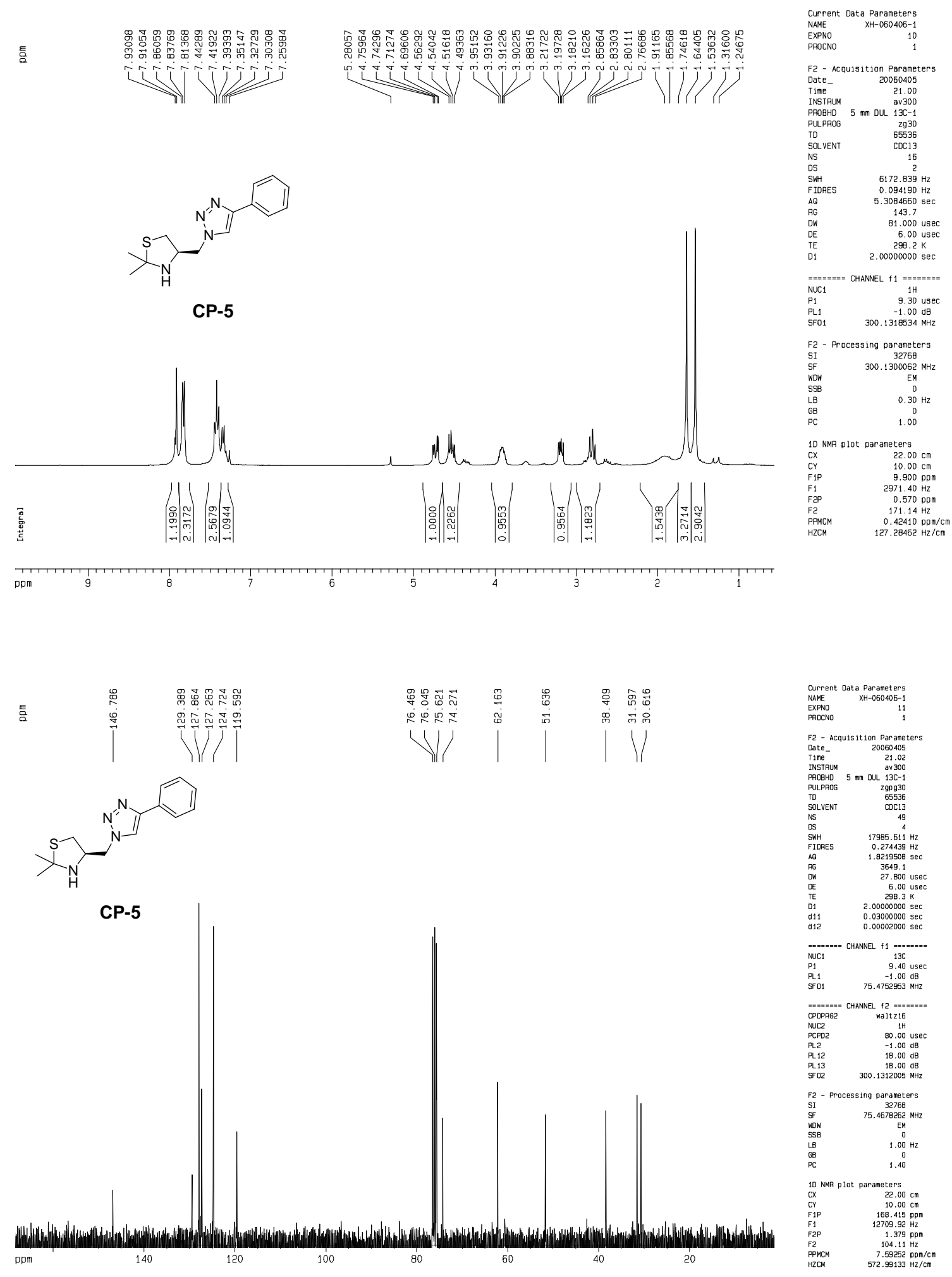

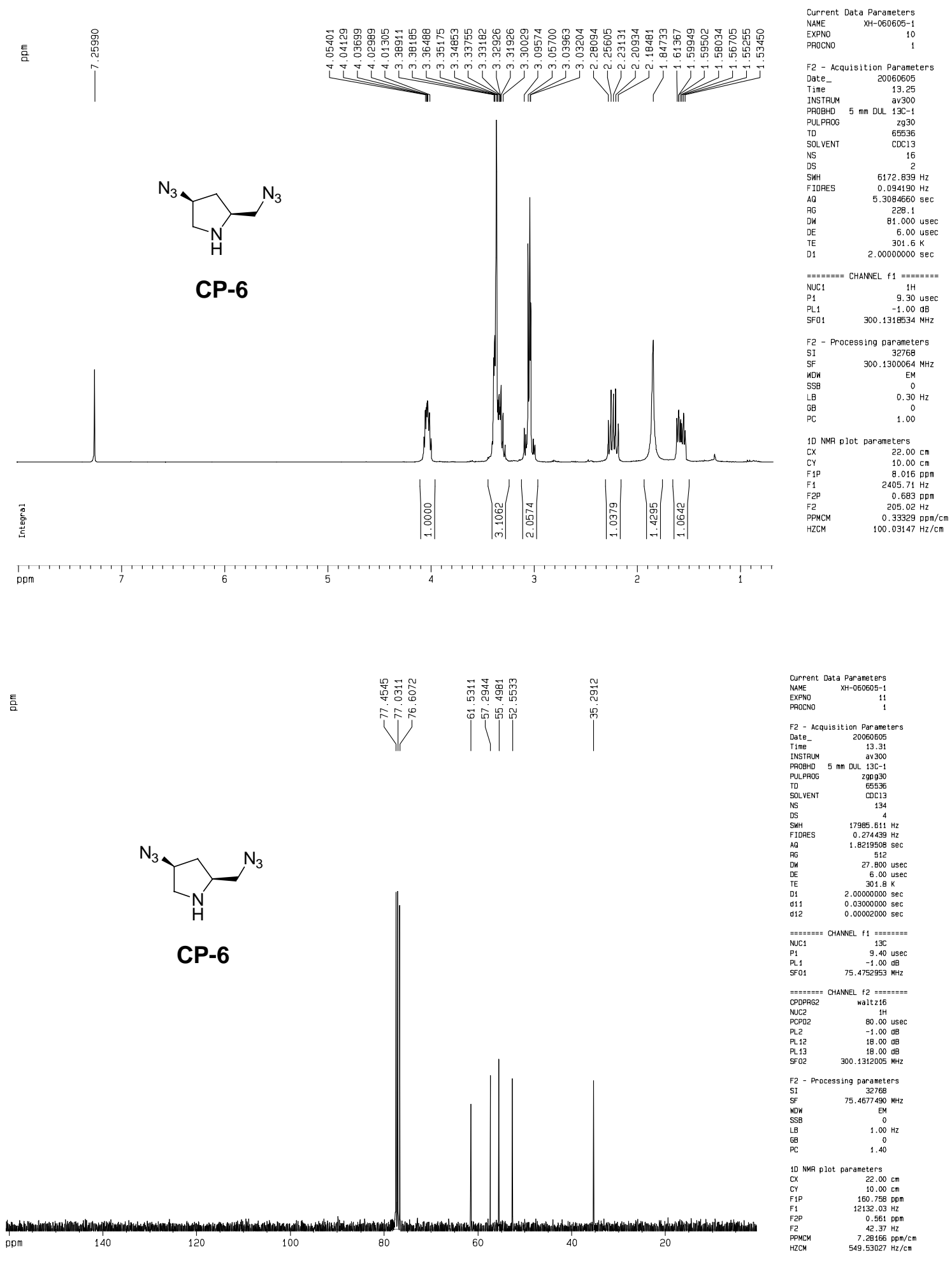


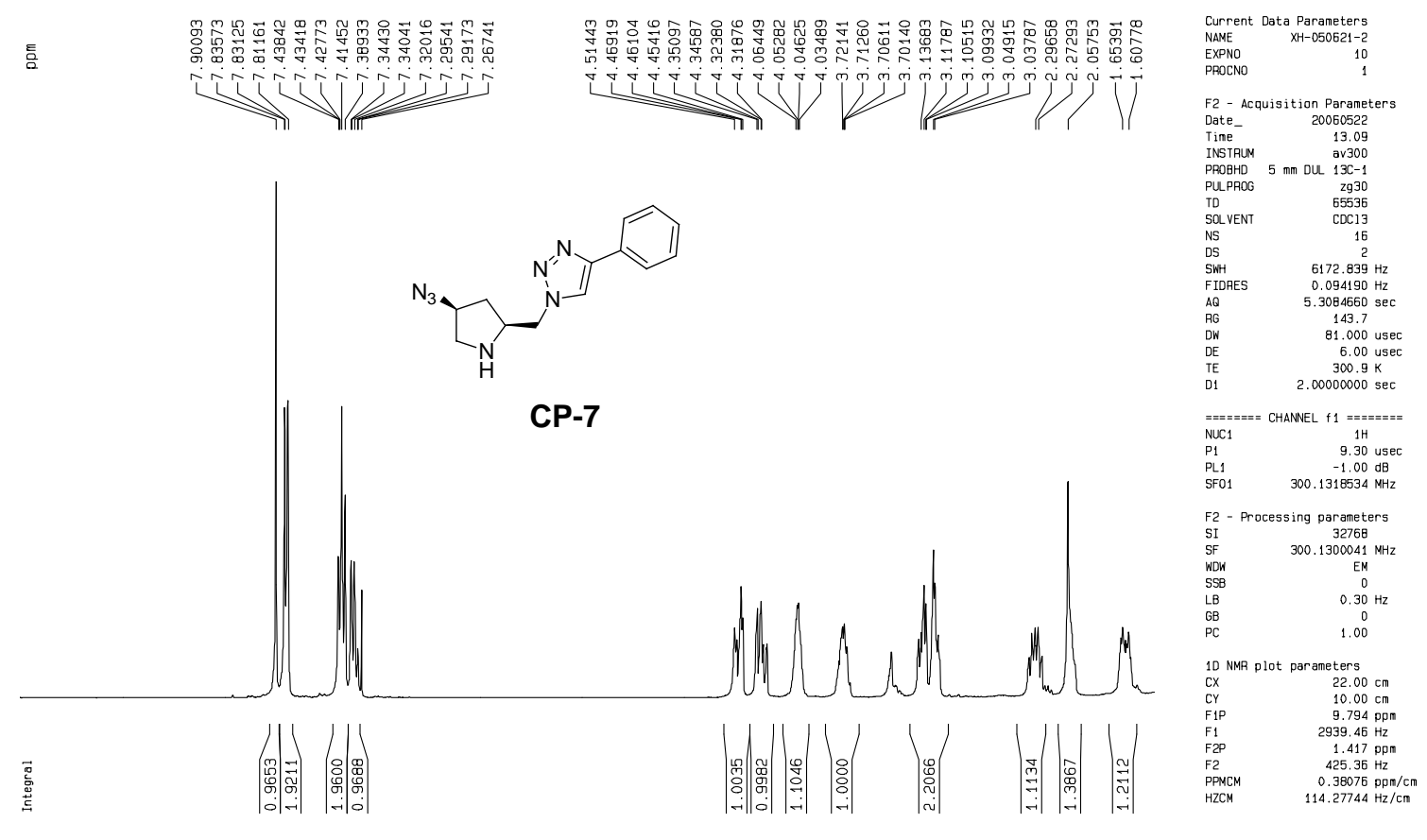

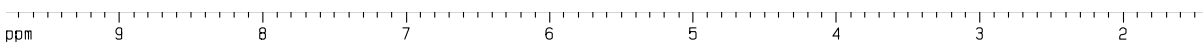

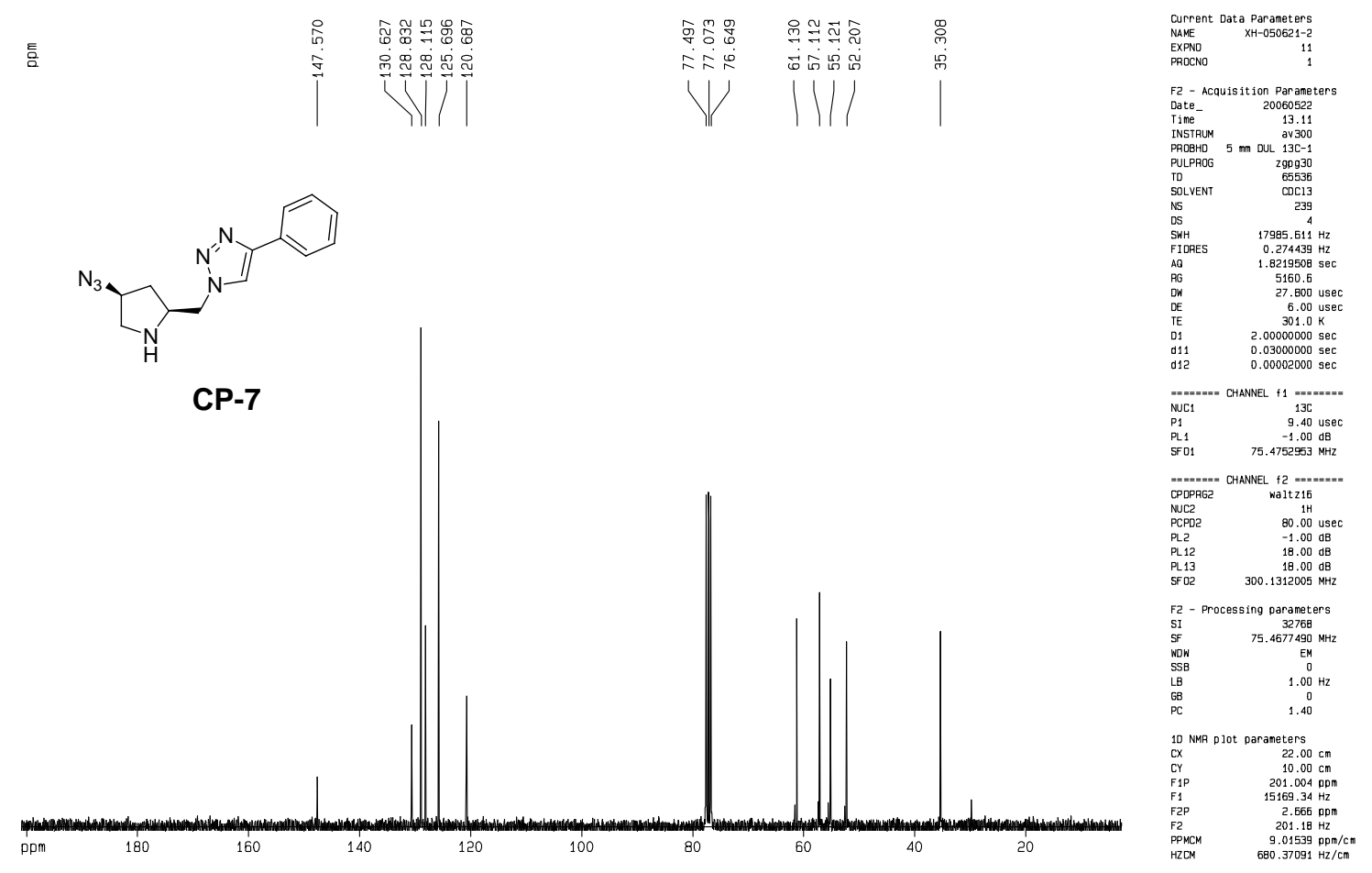



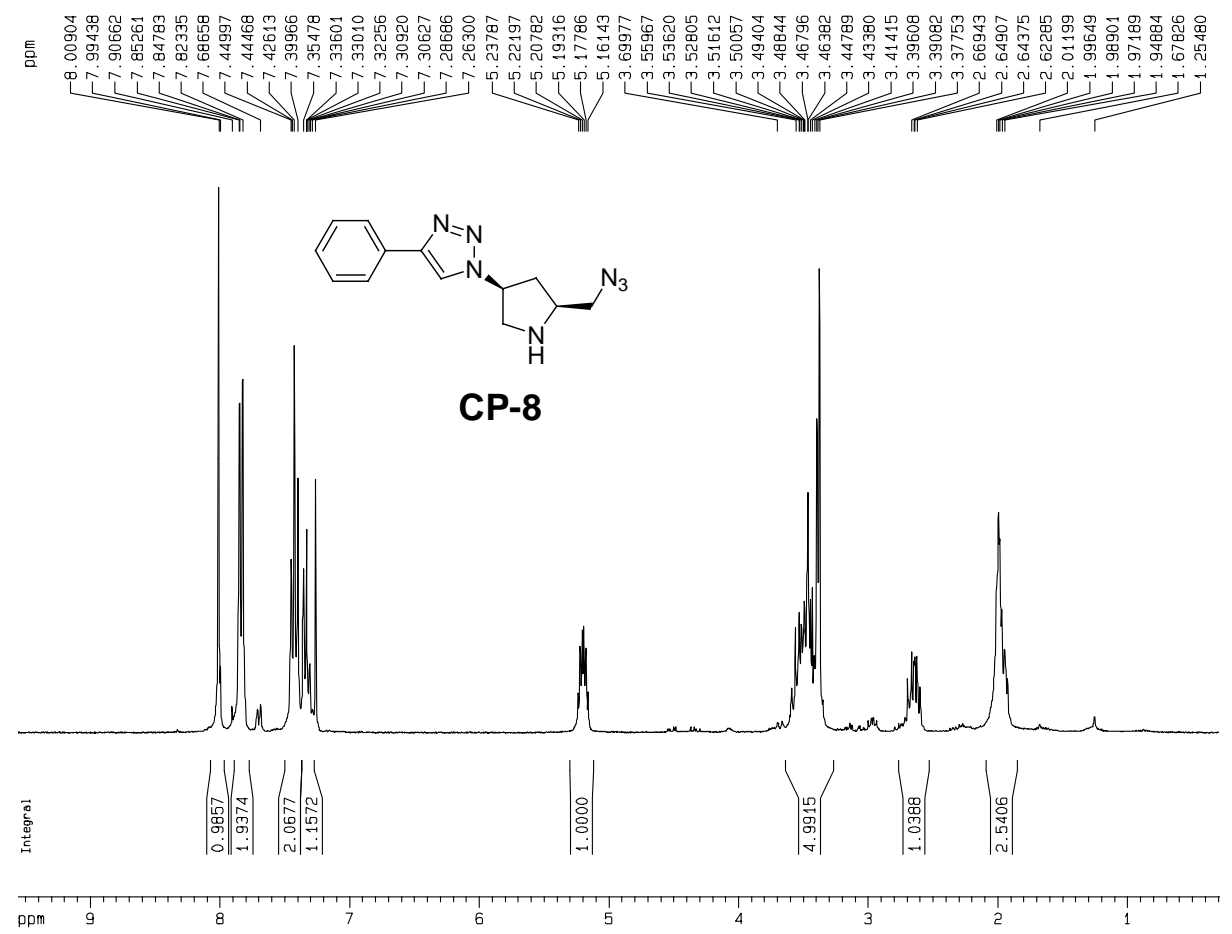

틈
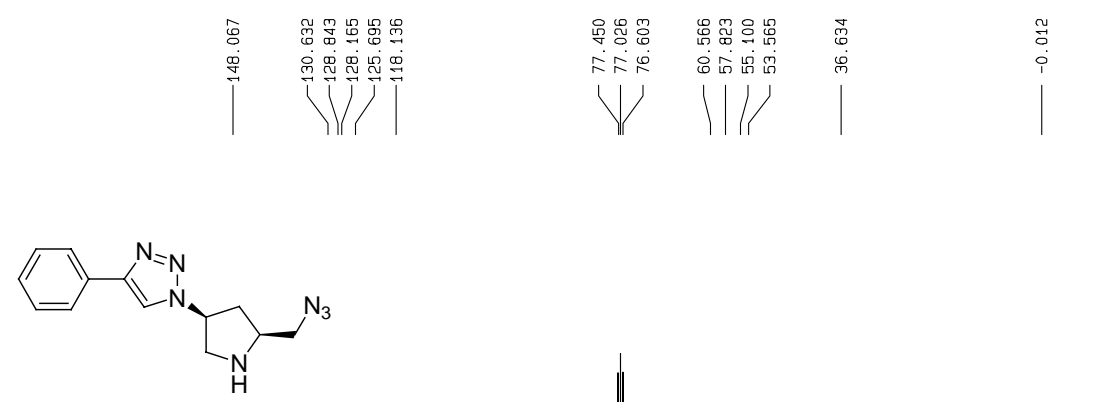

CP-8
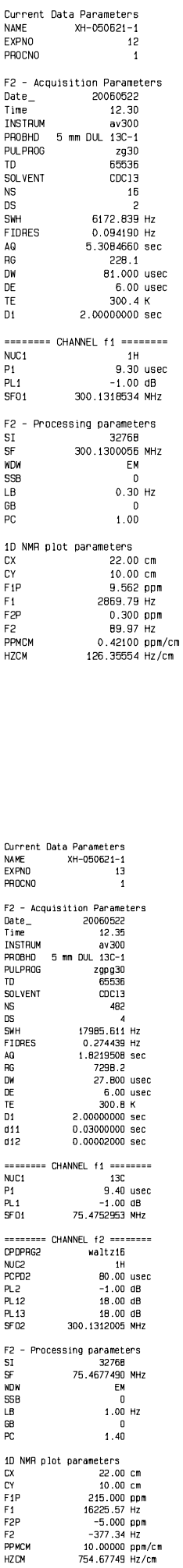


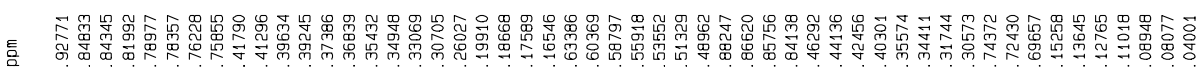

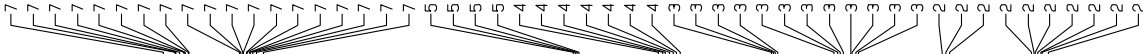
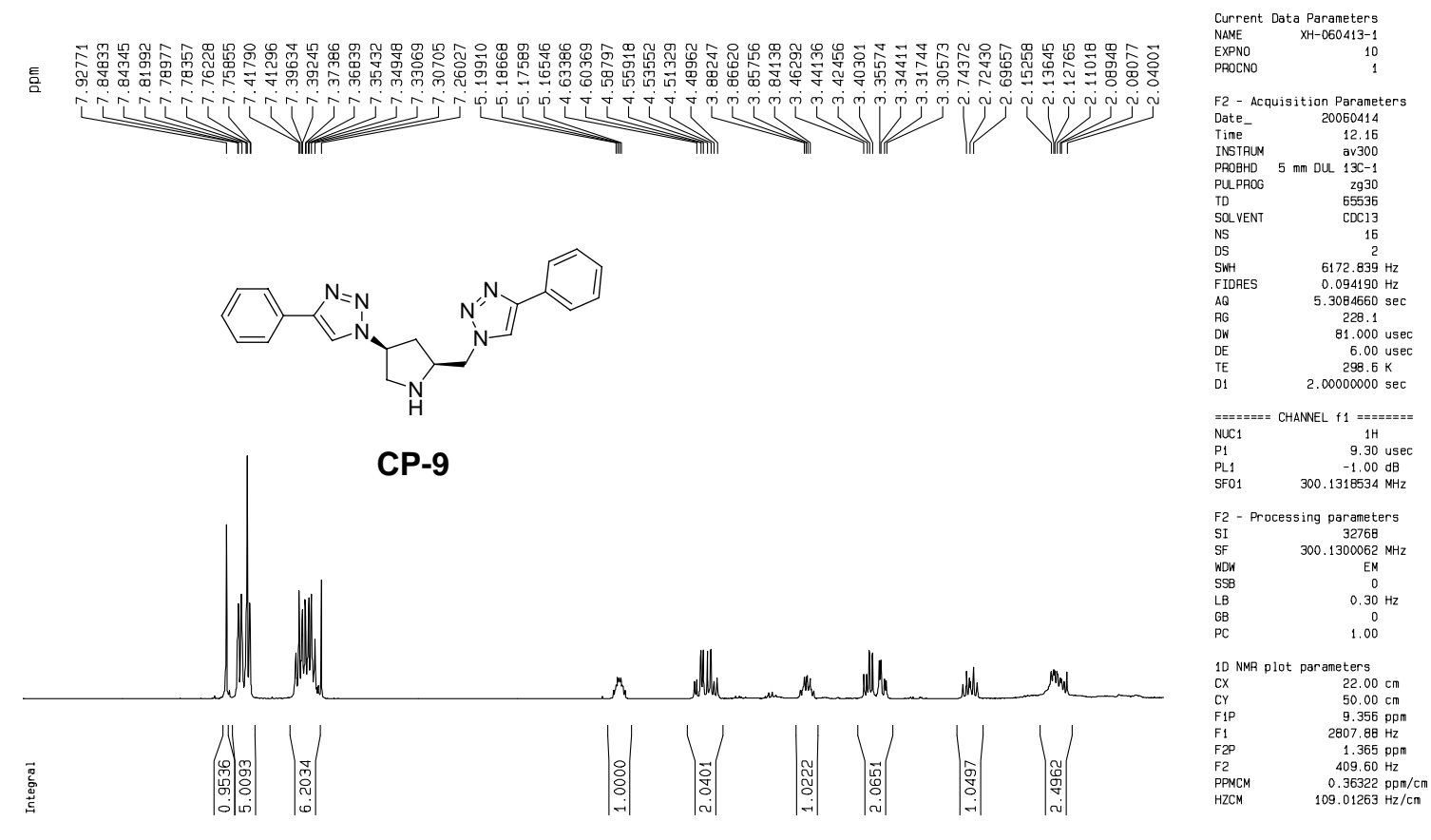

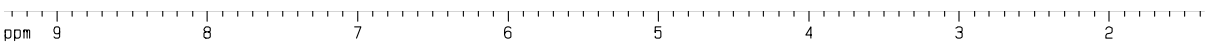

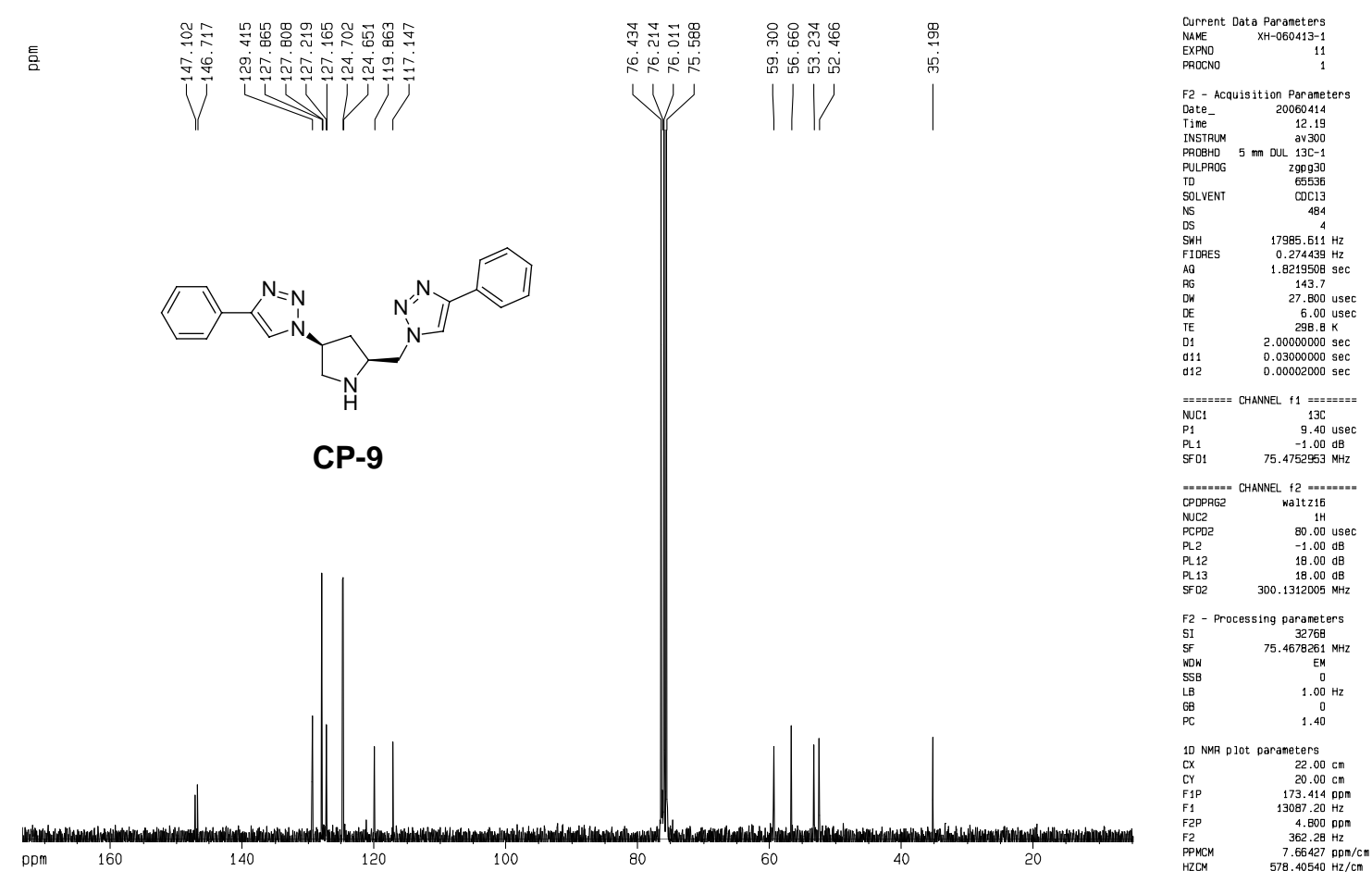



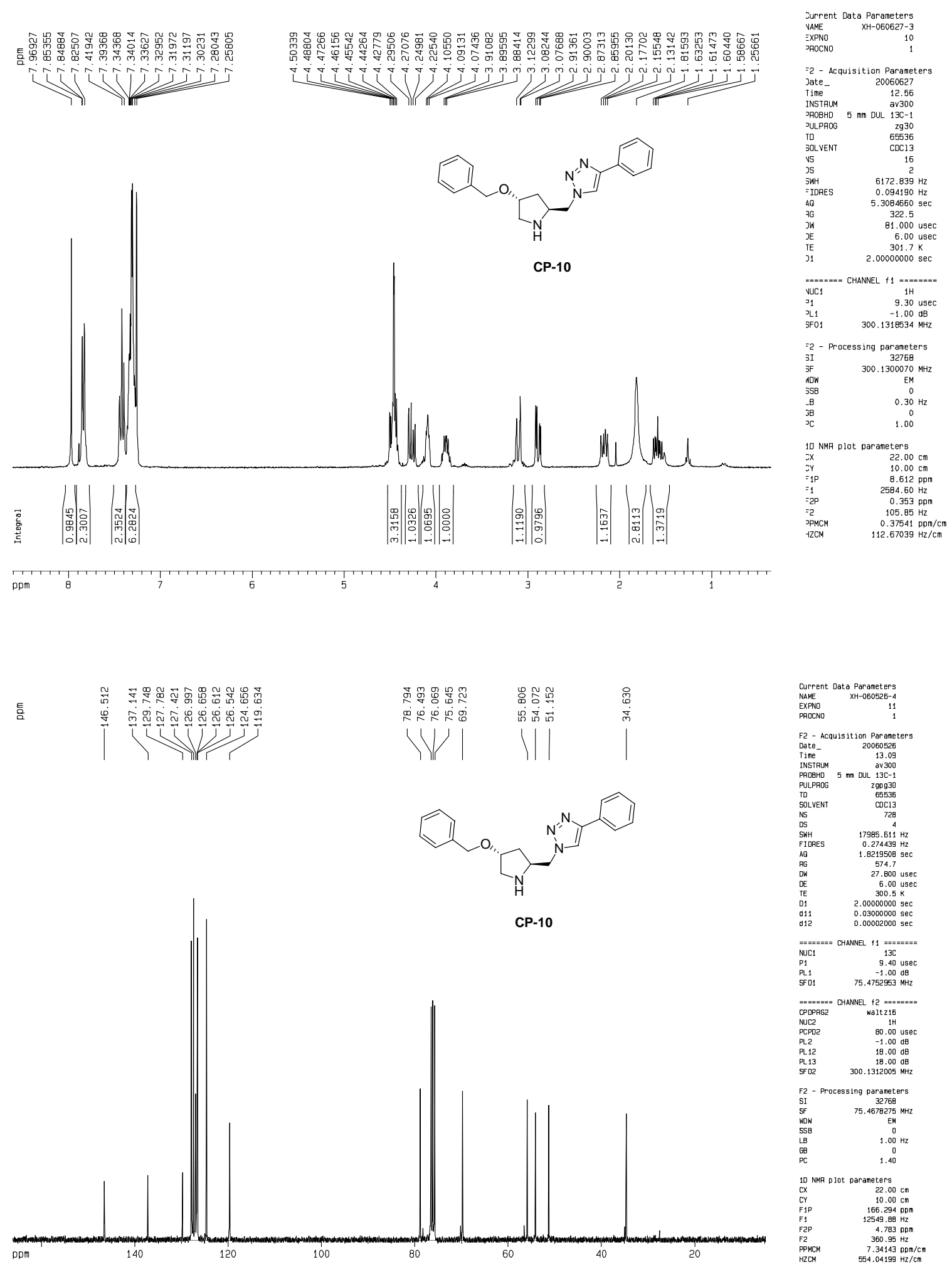

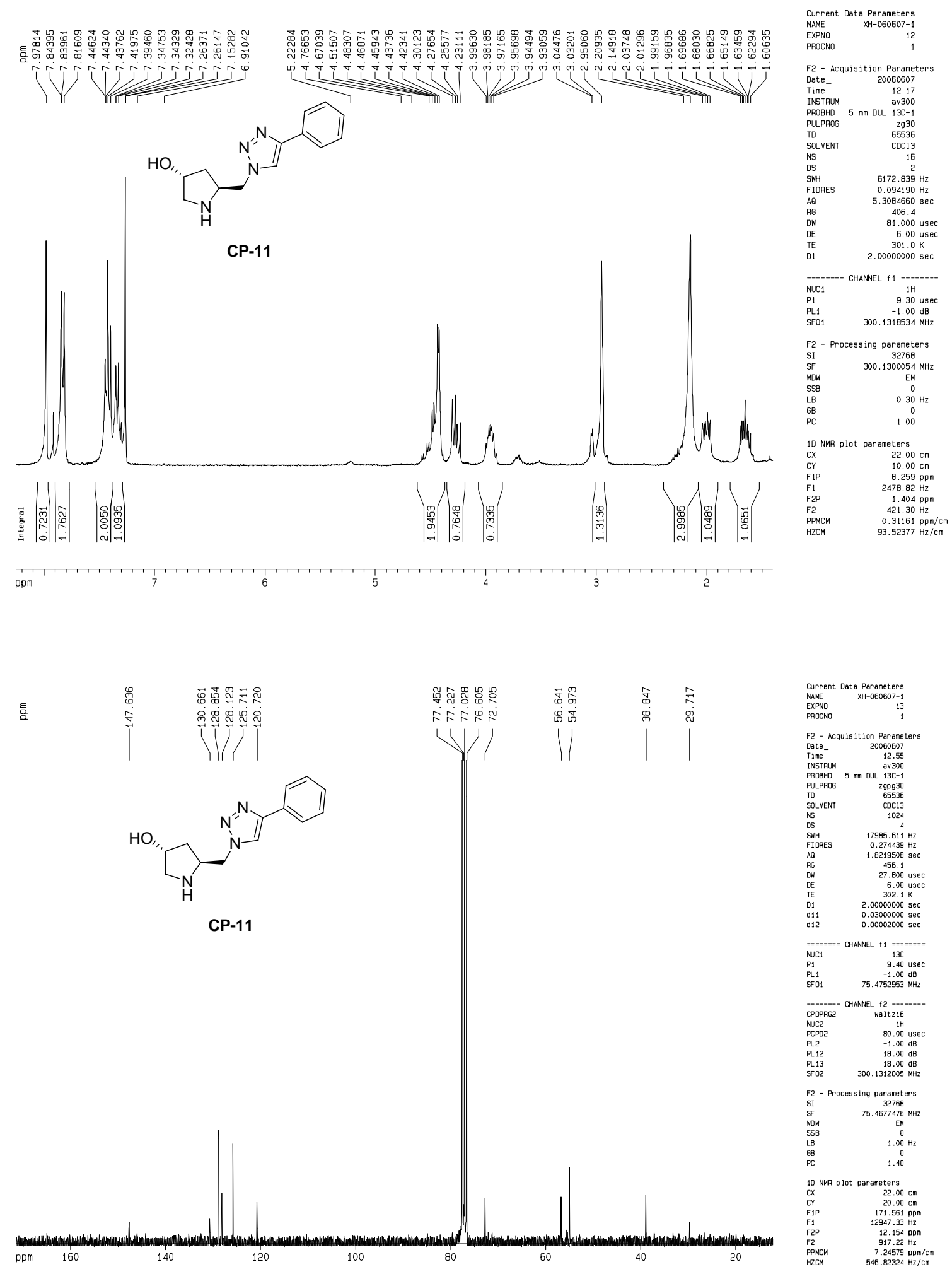

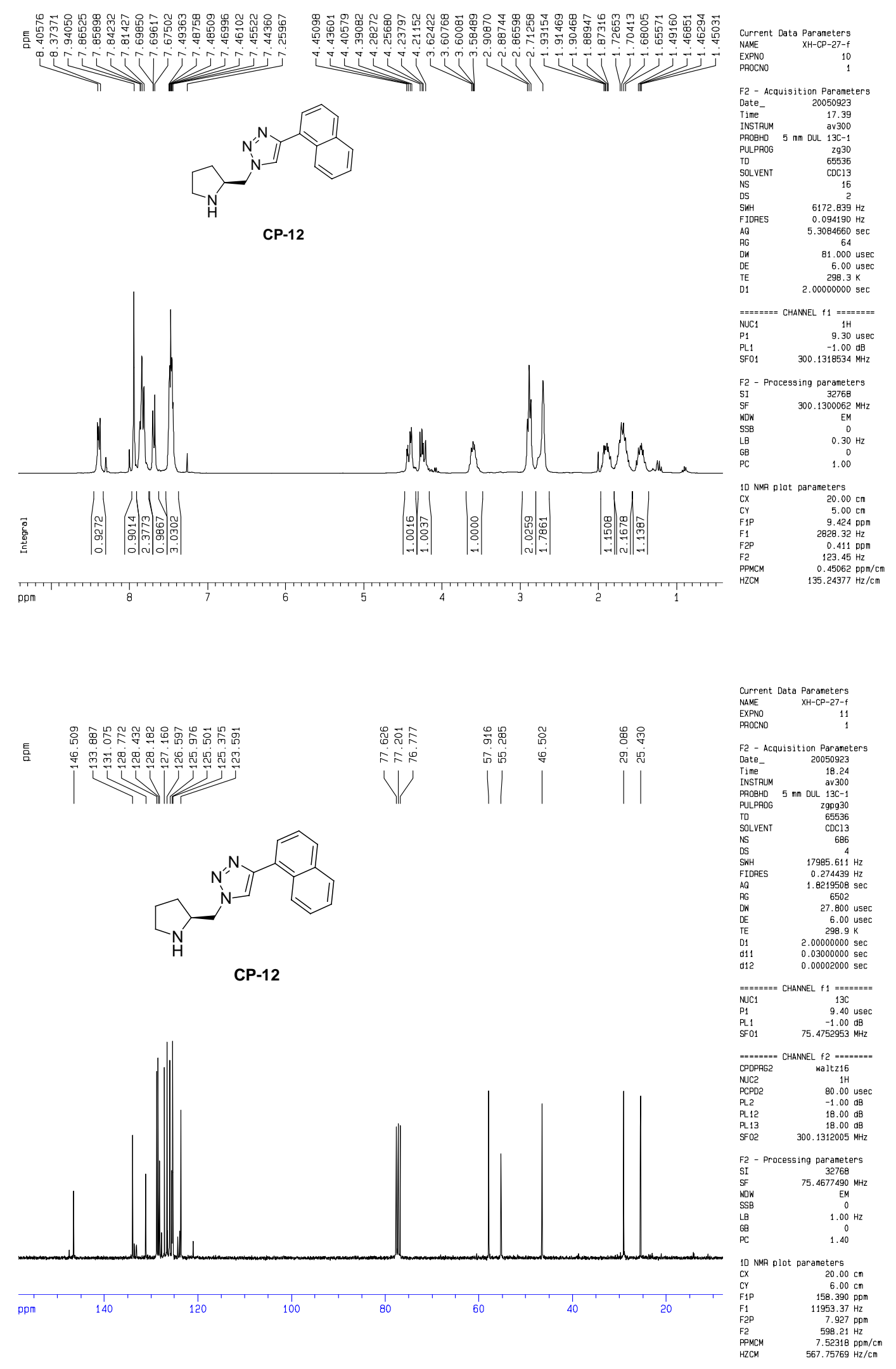

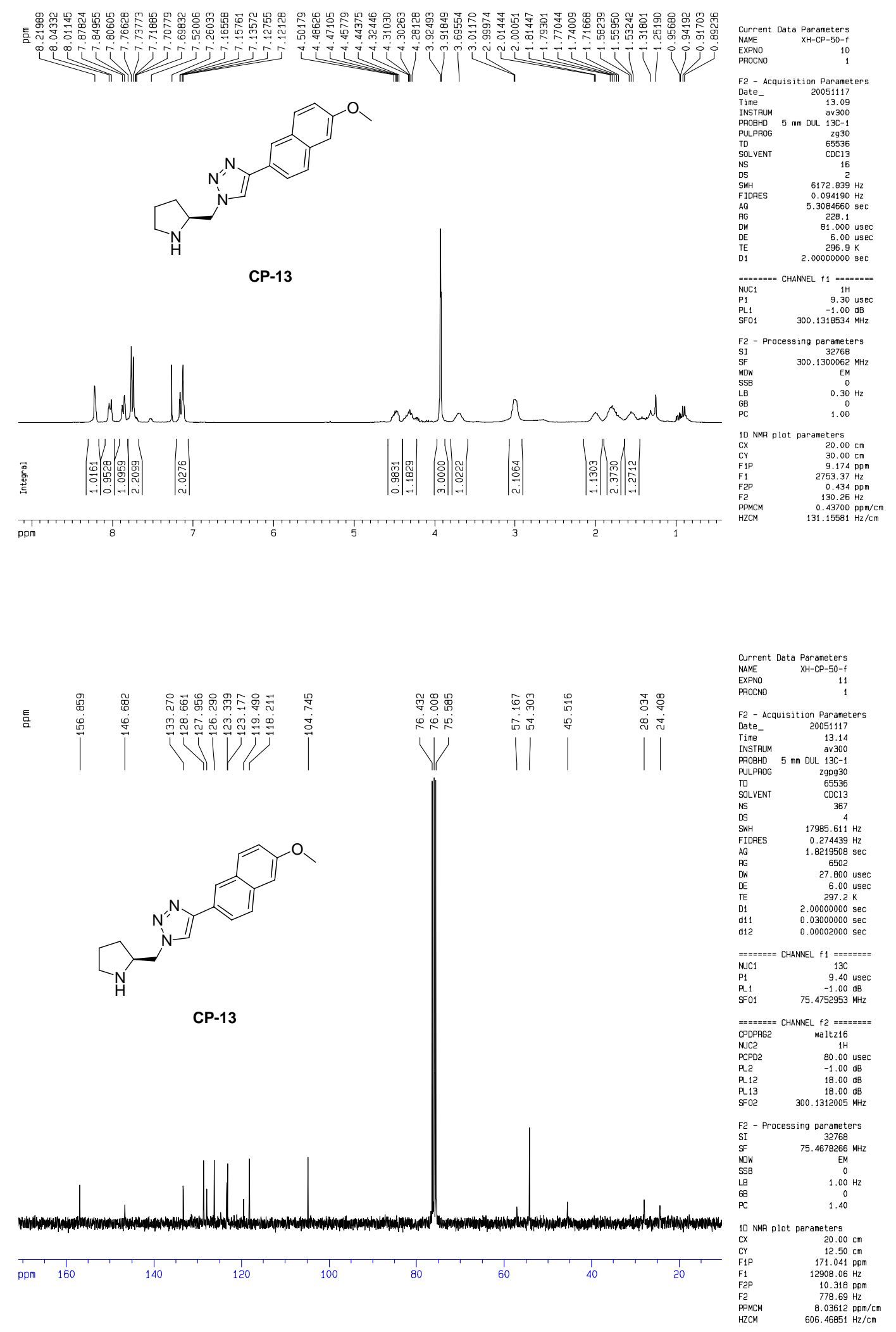

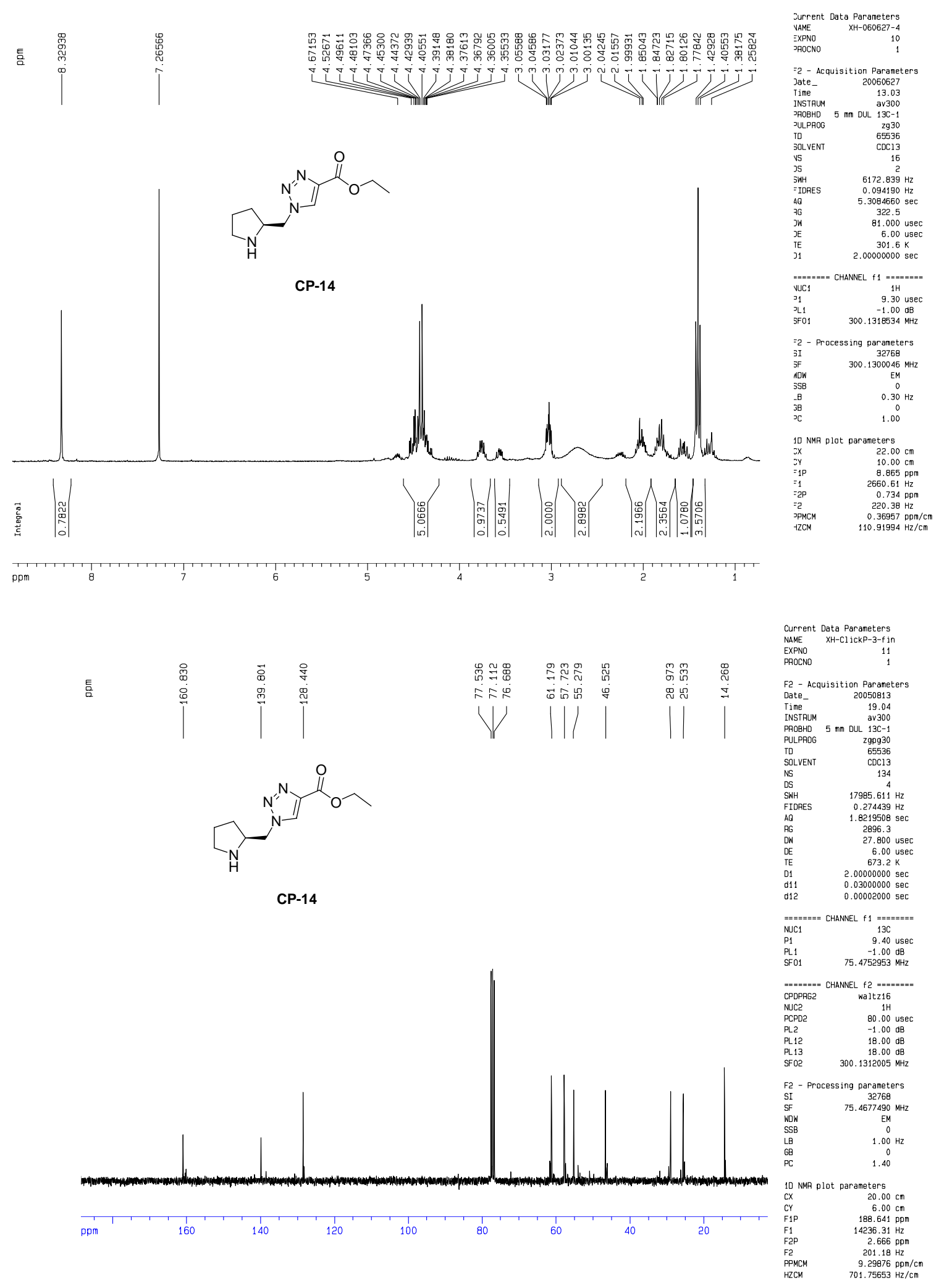

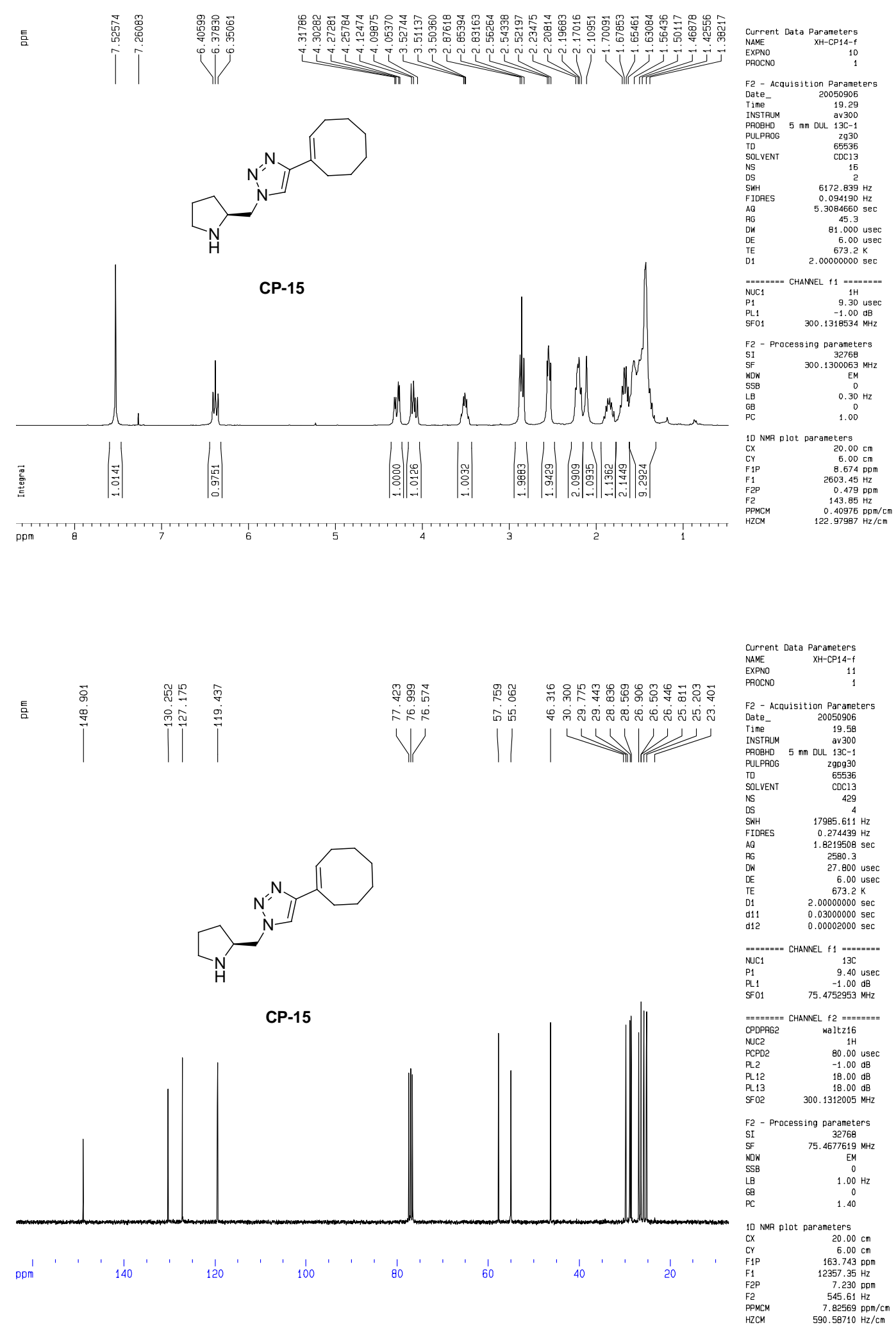

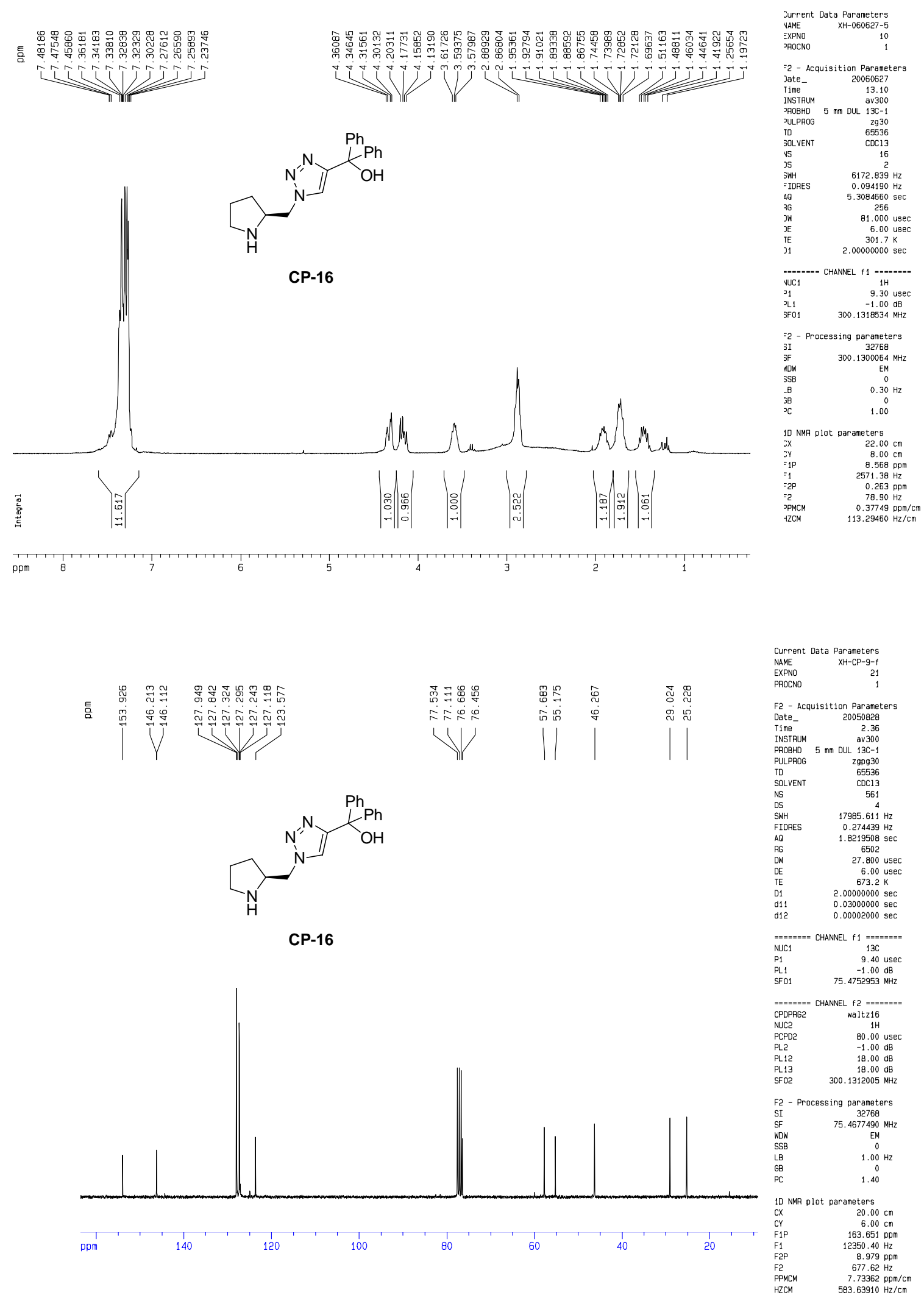

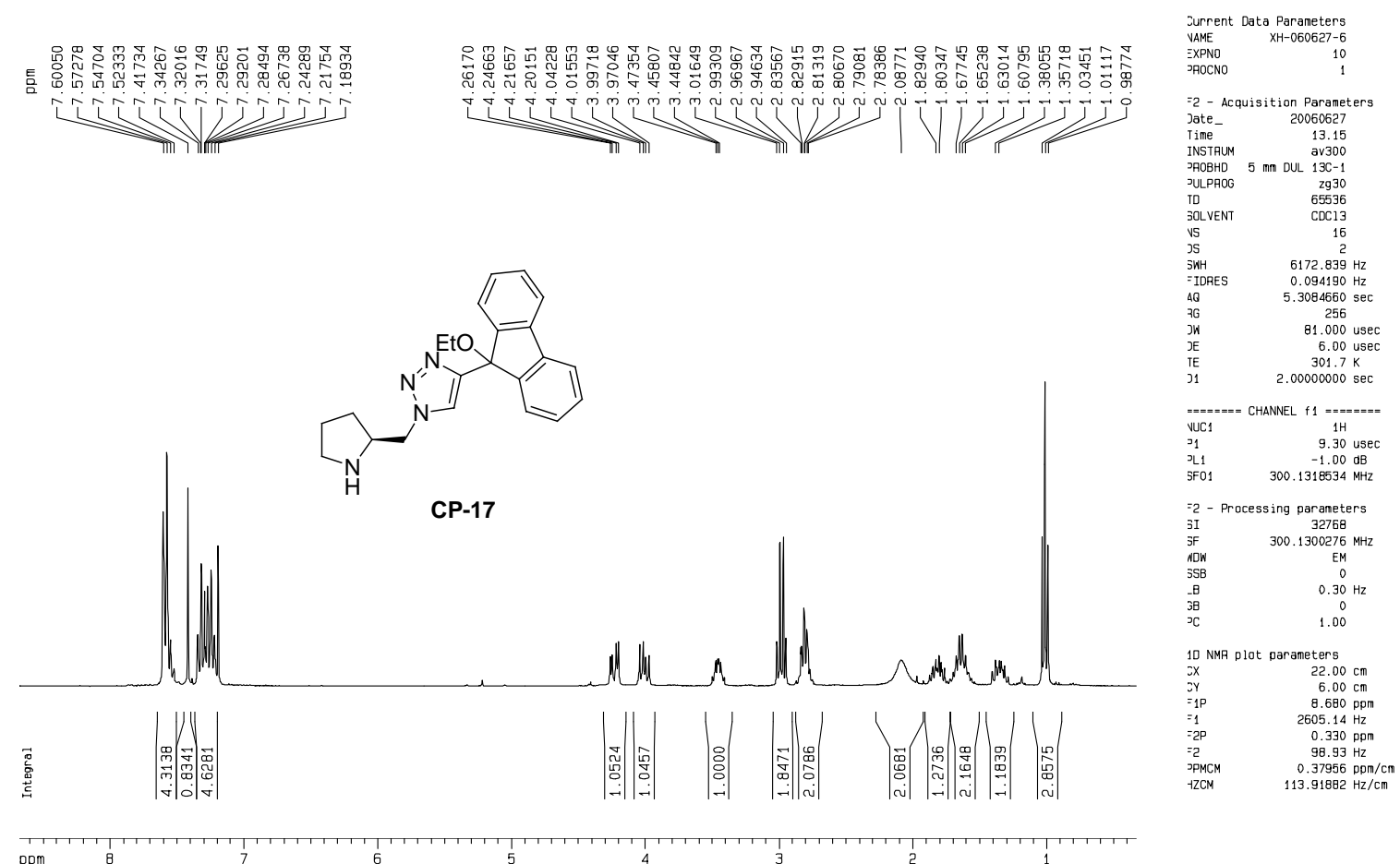

$$
\text { ppm }
$$

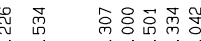

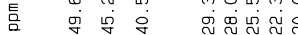

$\vec{\imath} \overrightarrow{\mid} \vec{l}$
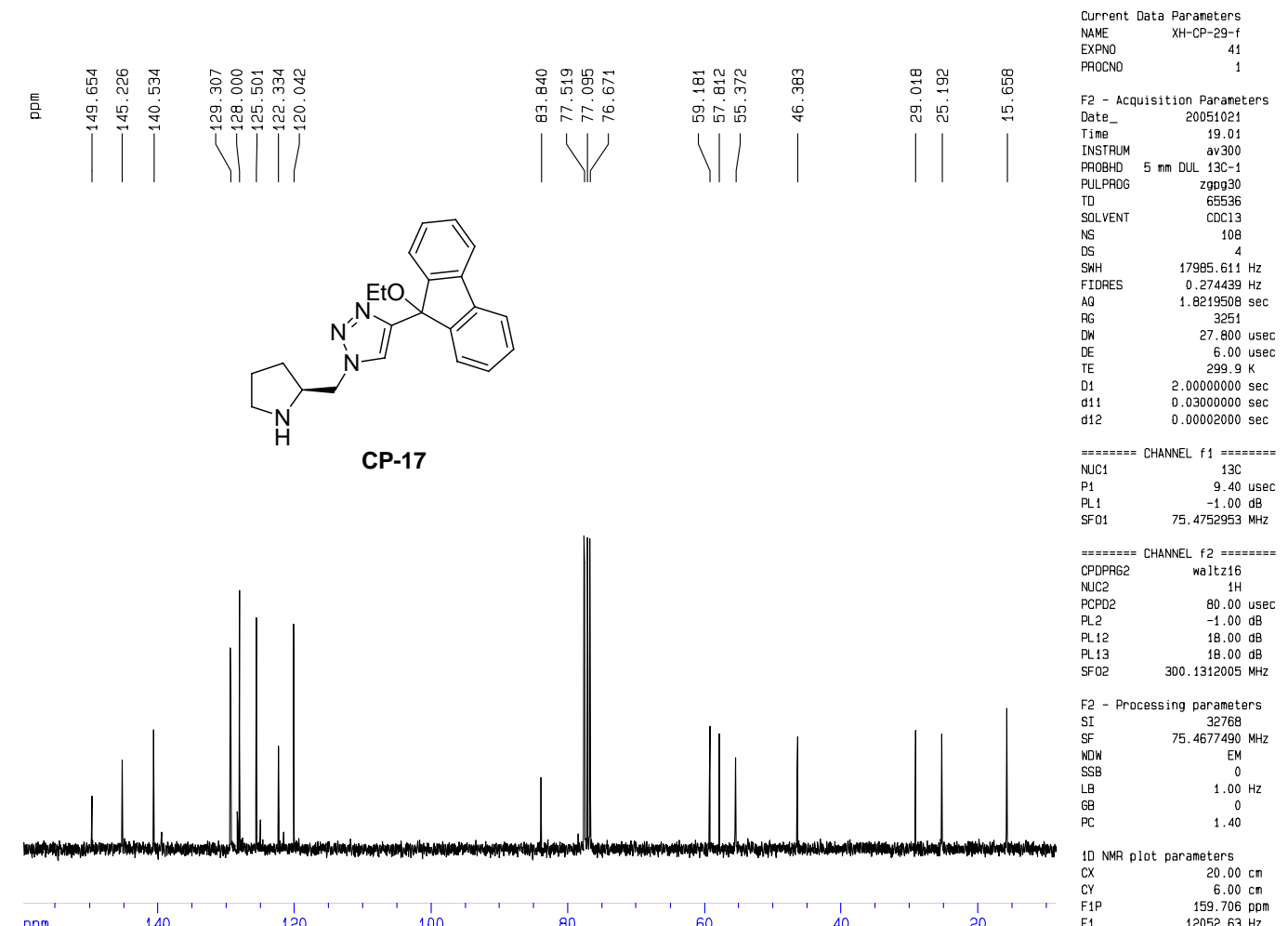

ppm ' '

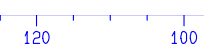

100

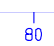

1
60
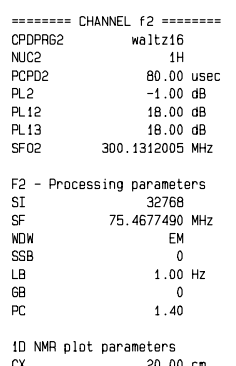

$c x$
$c y$
$c$
cy

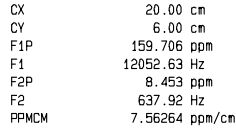



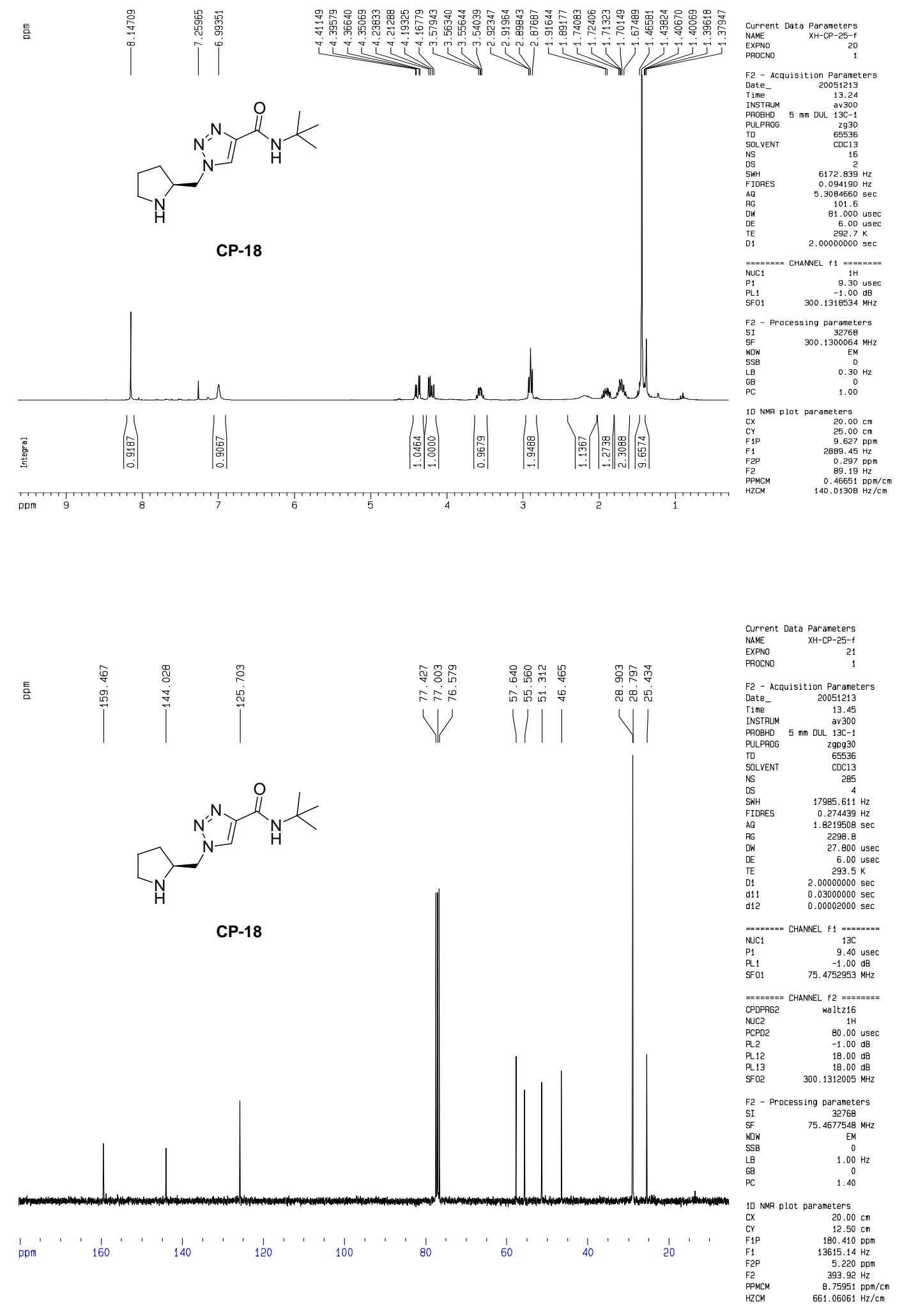

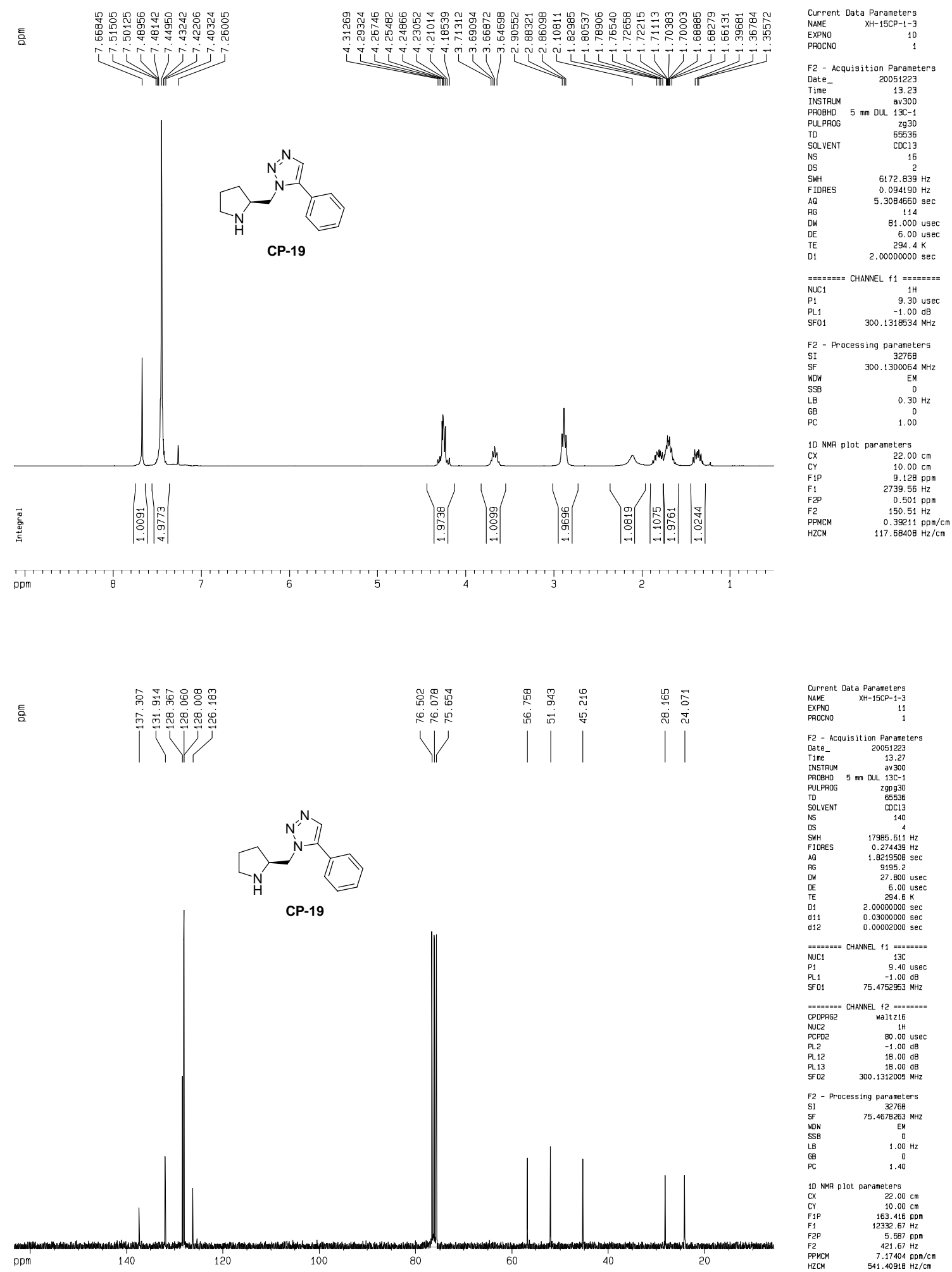


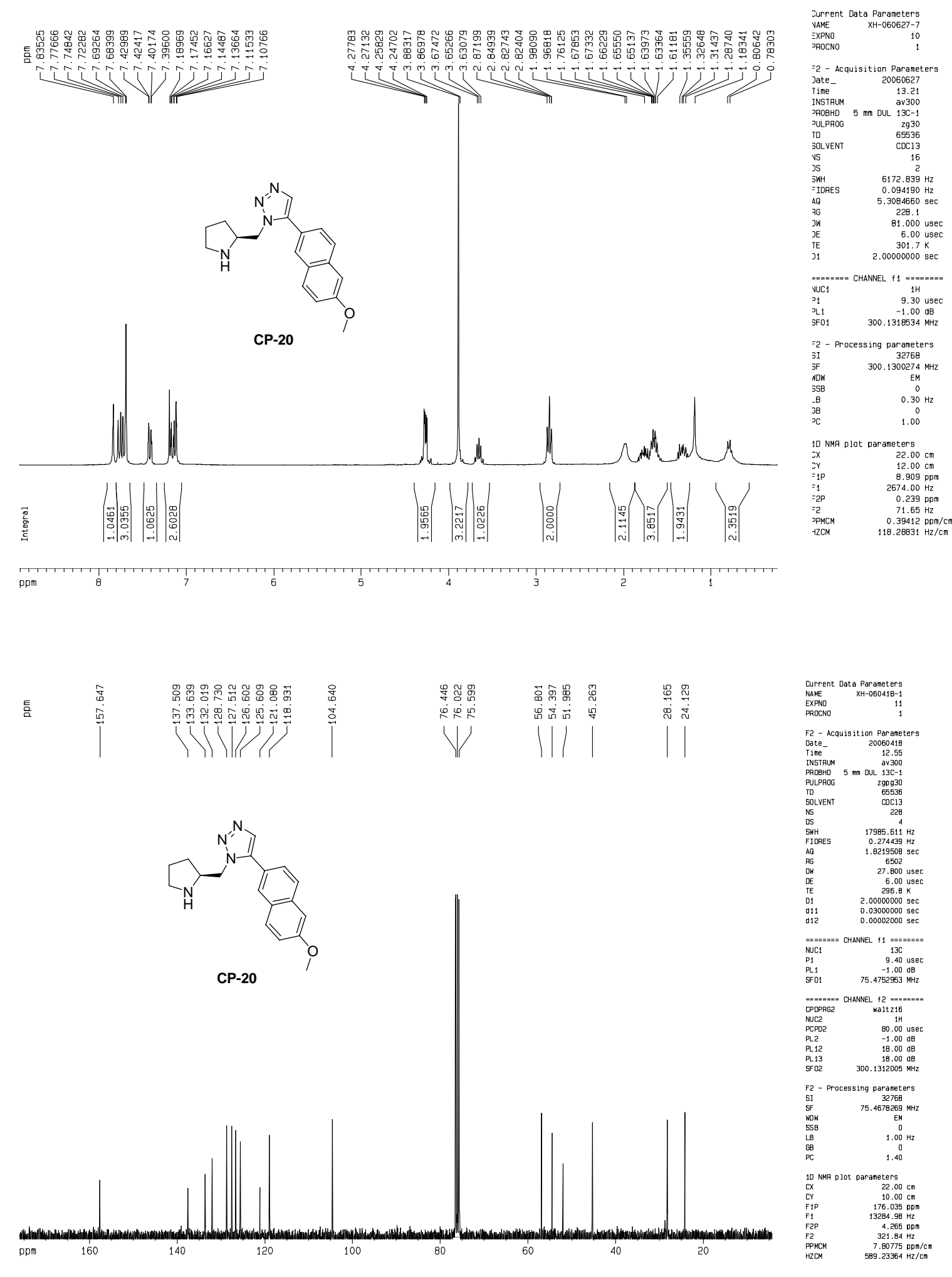




\section{${ }^{1}$ H NMR spectra for the Michael products}
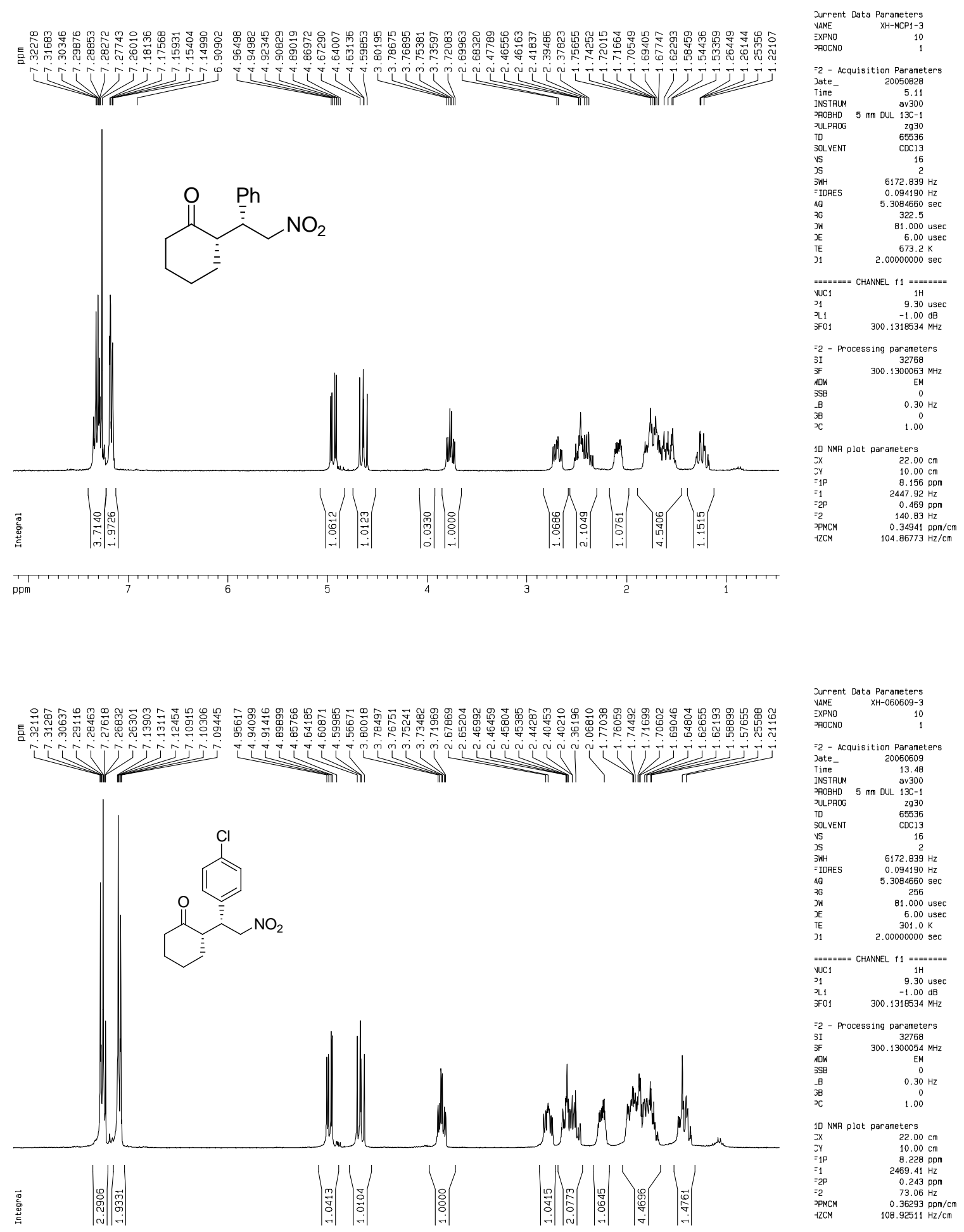

ppm
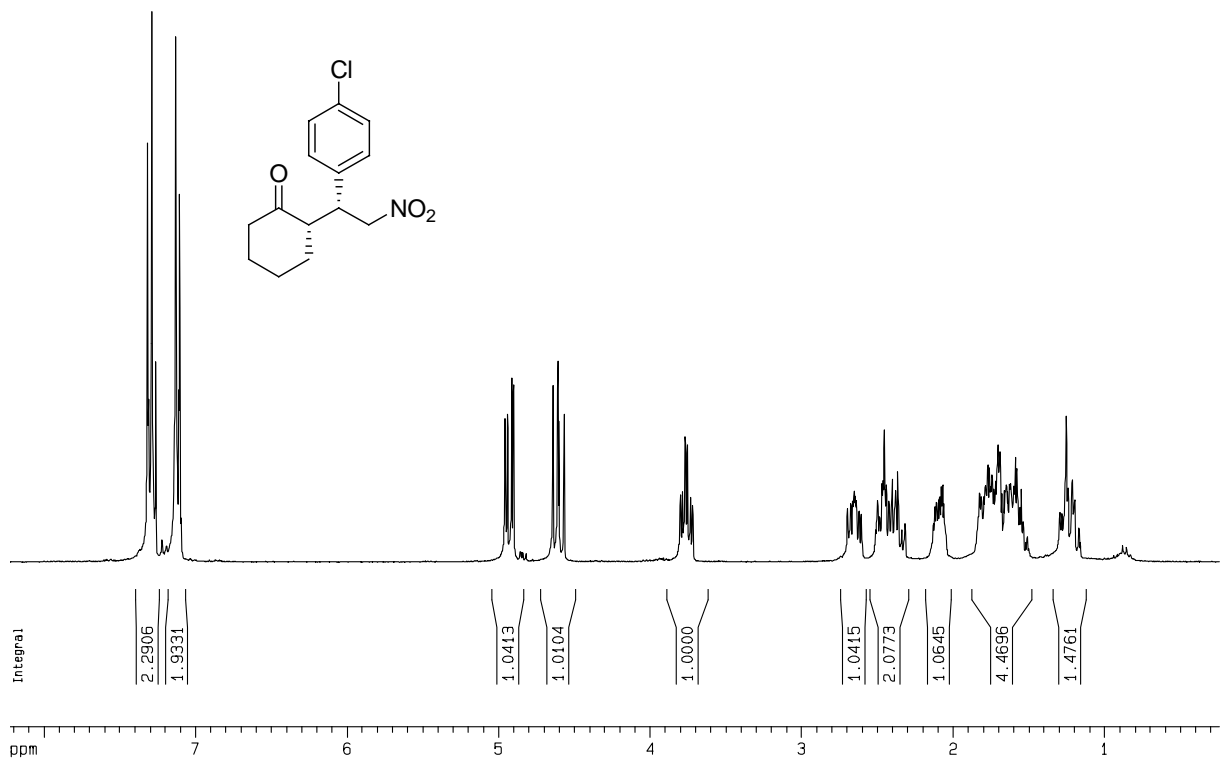

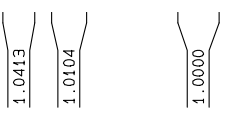
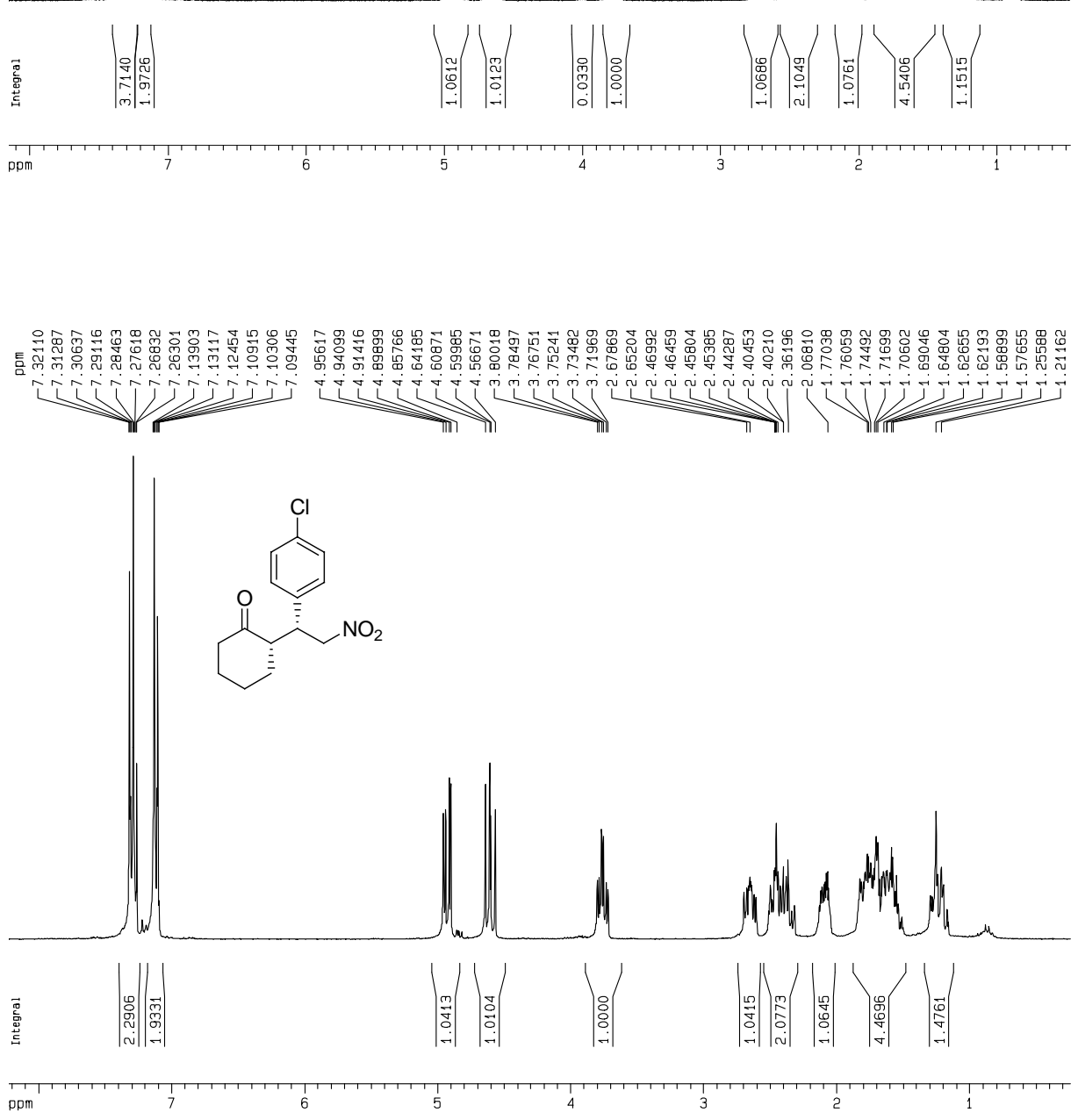

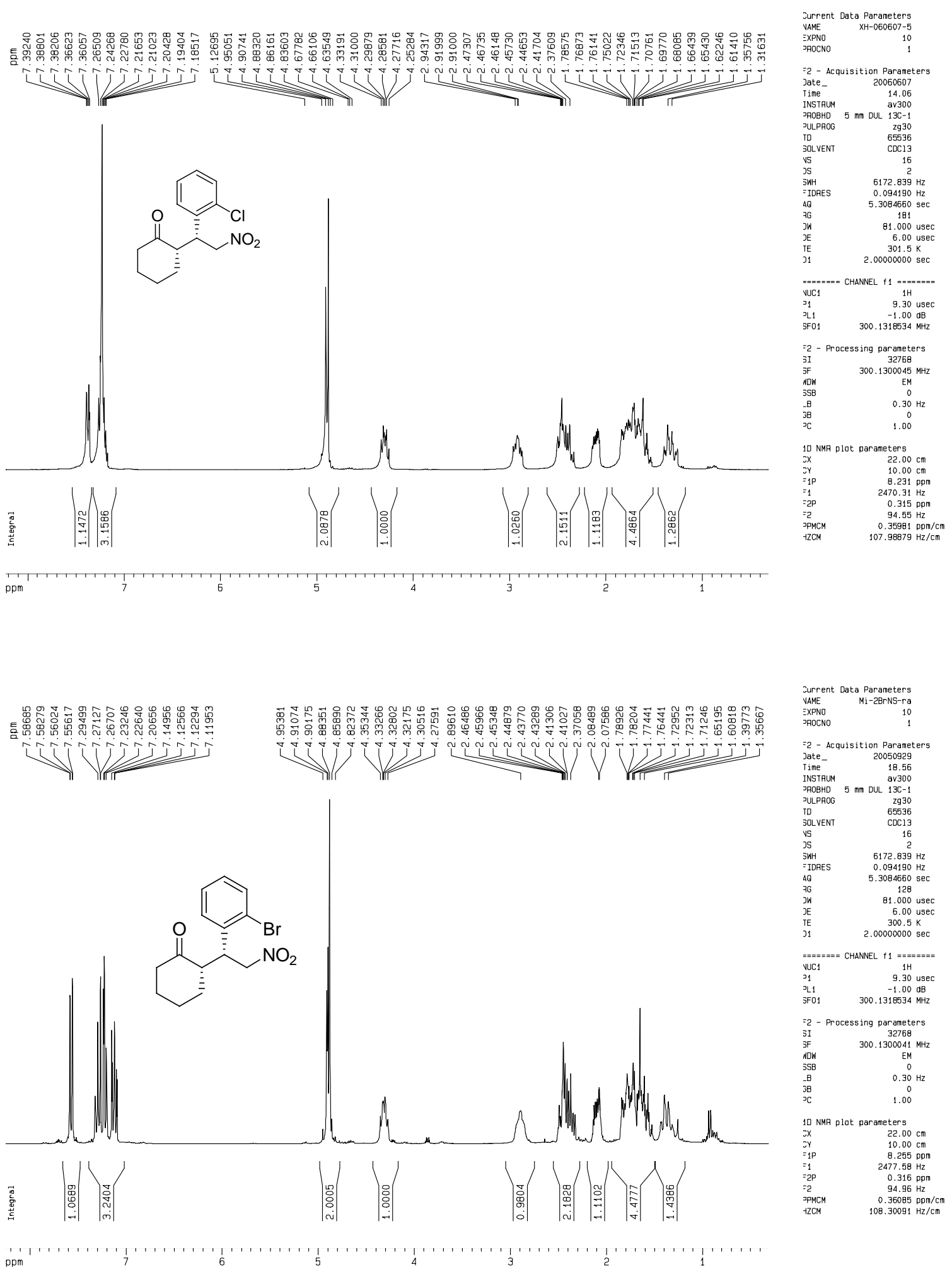

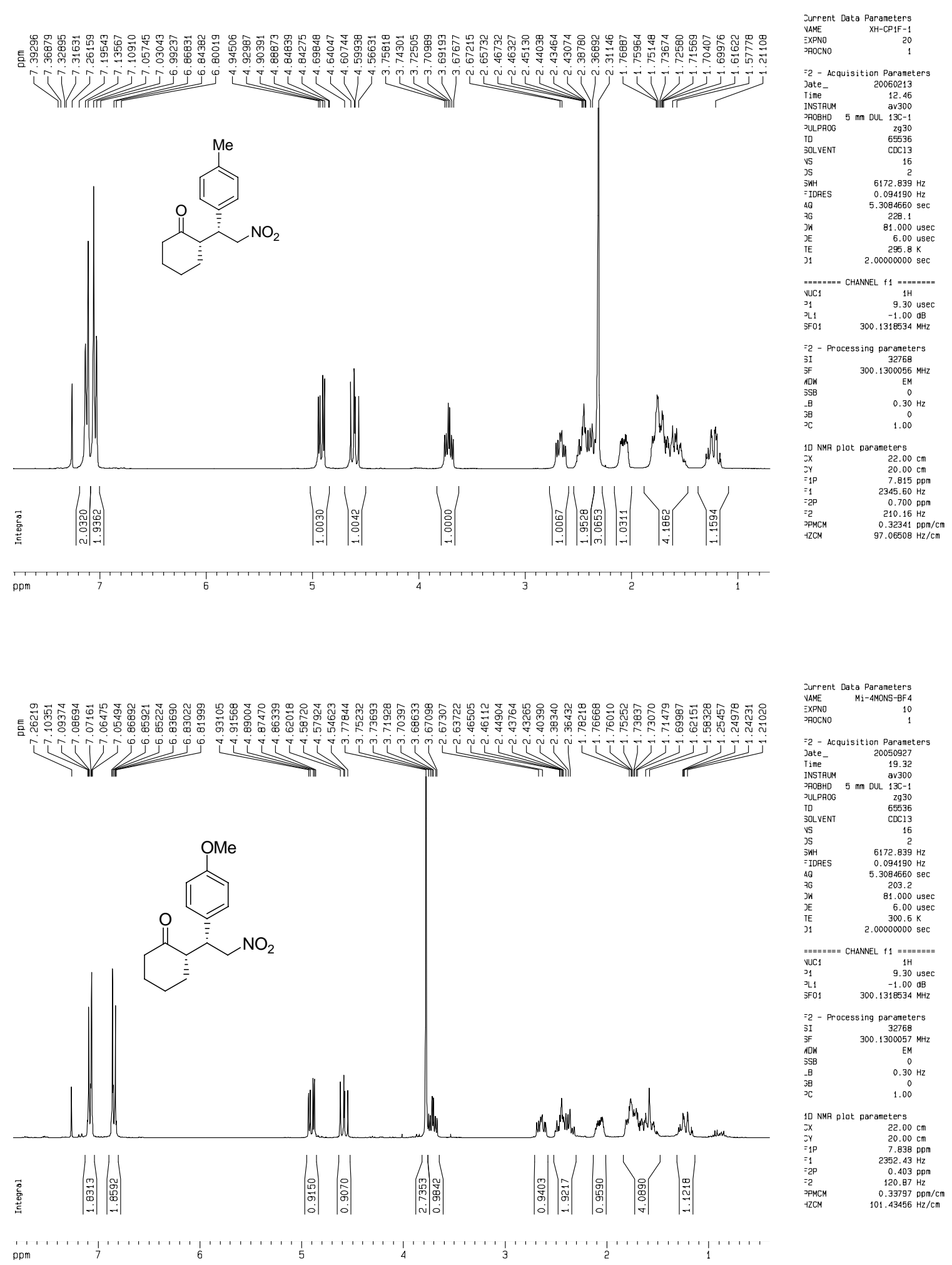

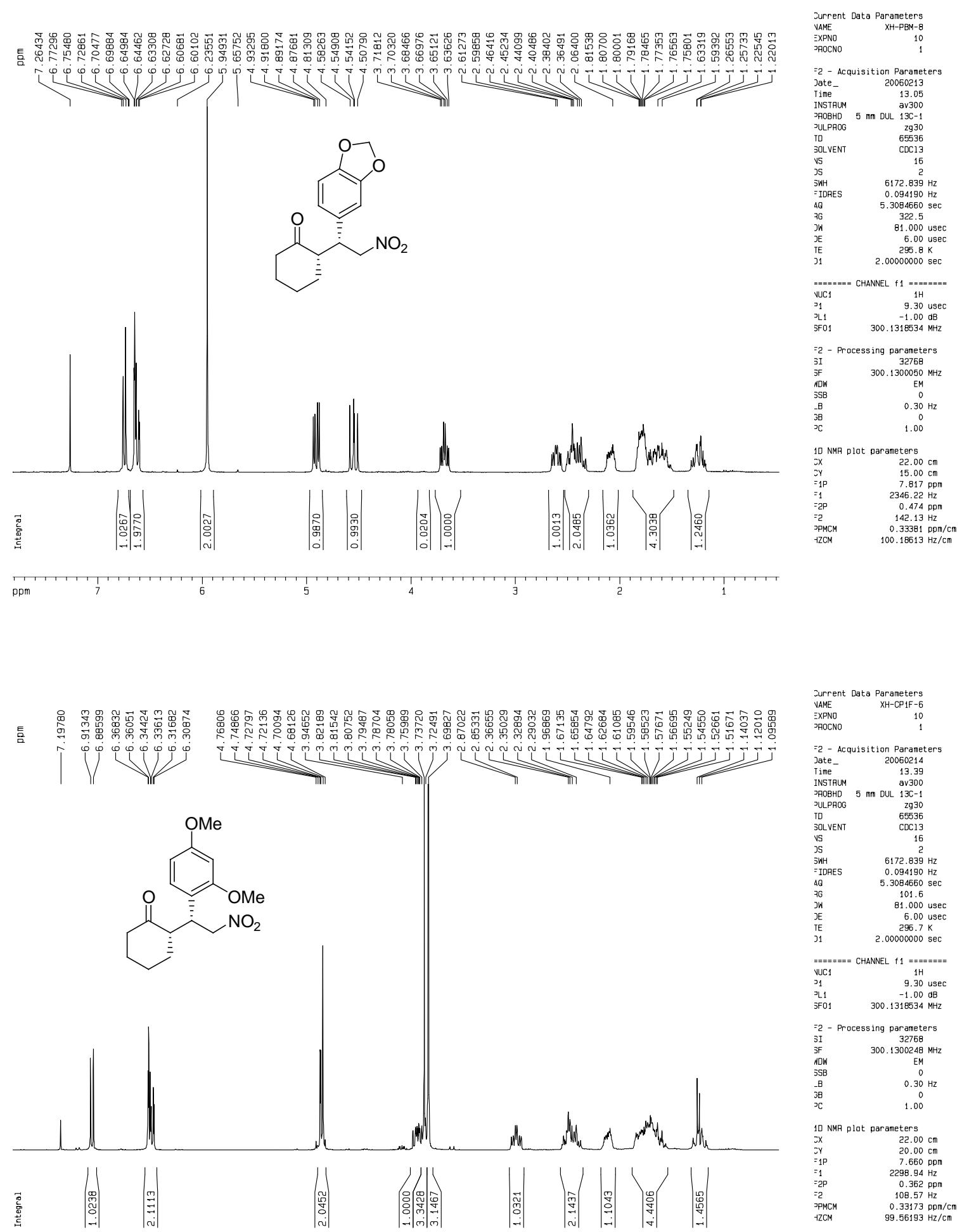

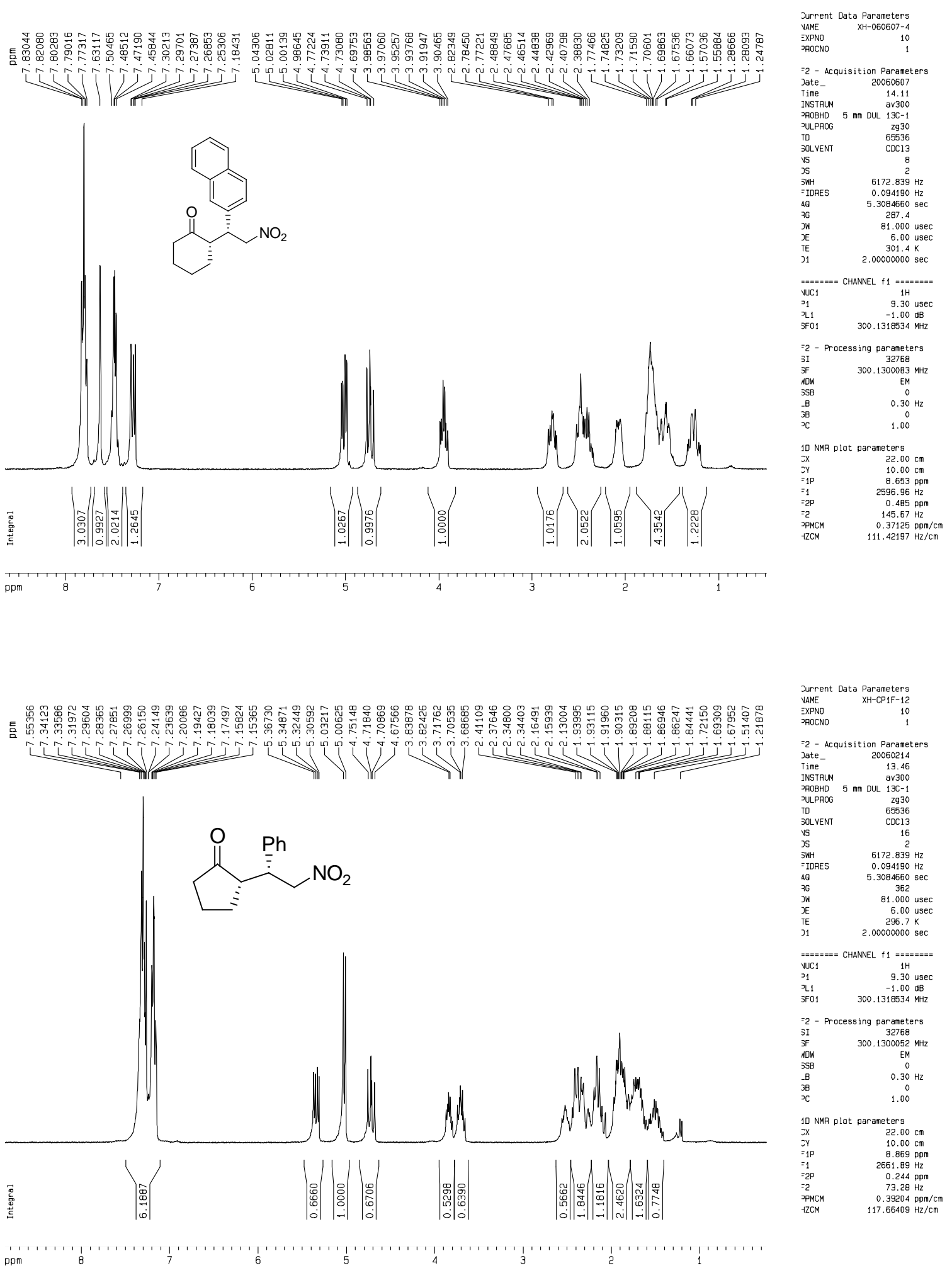

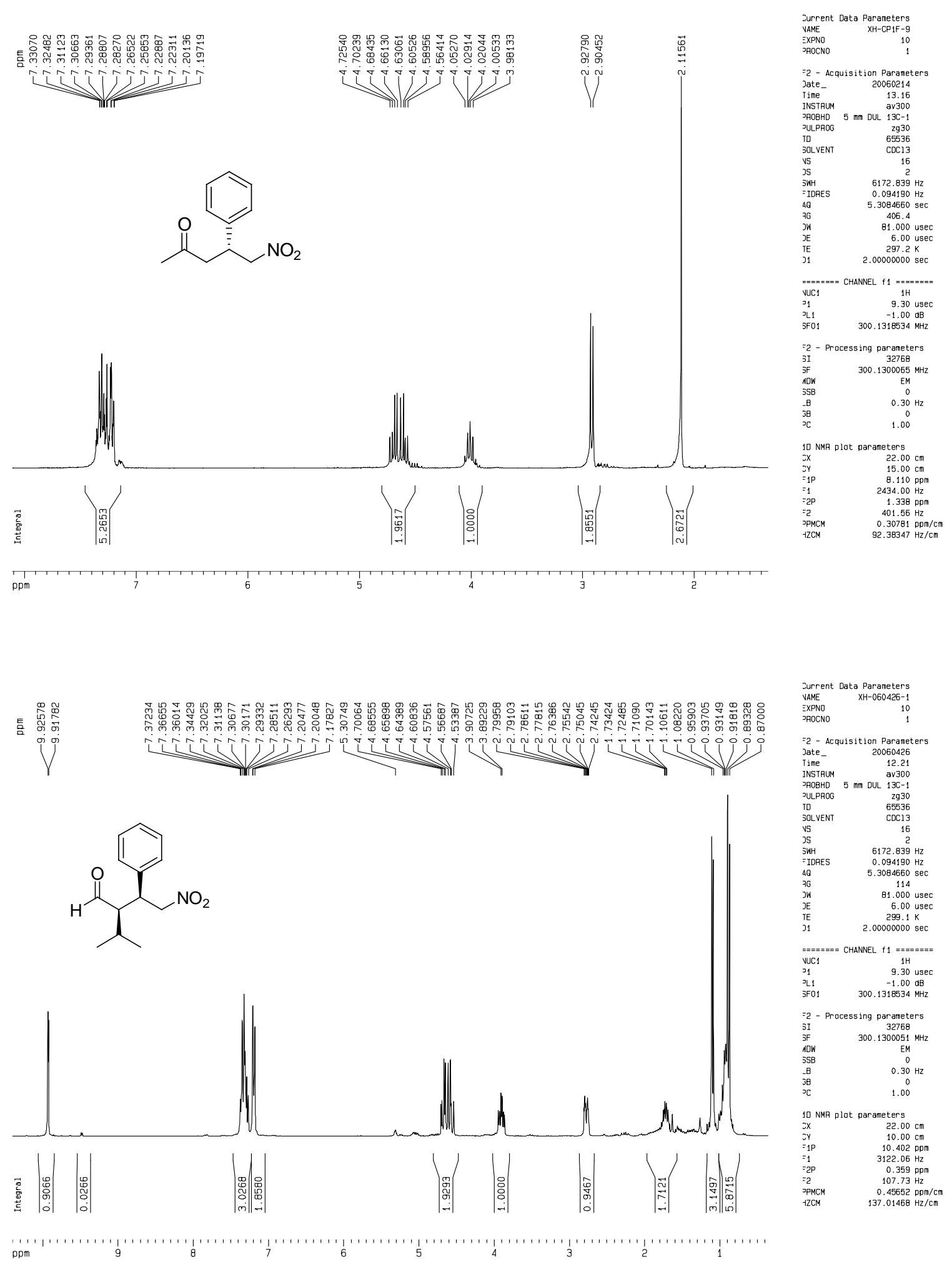